\title{
Flow Over a Flat Plate with Uniform Inlet and Incident Coherent Gusts
}

\author{
IMRAN A F G A N $\mathbf{N}^{1,2} \dagger, \quad$ S OFIANE BENHAMADOU C H E $\mathbf{H}^{3,4}$ \\ X I N G S I H A N ${ }^{1}$ \\ PIERRE SAGAUT ${ }^{1}$ AND DOMINIQUE LAURENCE \\ ${ }^{1}$ Institut Jean Le Rond d'Alembert, Université Pierre et Marie Curie-Paris VI, 4 place Jussieu \\ - case 162, 75252 Paris, France \\ ${ }^{2}$ Modelling \& Simulation Centre, School of MACE, University of Manchester, M13 9PL, UK \\ ${ }^{3}$ Mécanique des Fluides Energies et Environnement (MFEE), EDF - R\&D, 6 quai Watier, \\ 78401 Chatou, France \\ ${ }^{4}$ LaMSID, UMR CNRS EDF 2832, Clamart, France
}

(Received xx xxxx 2012 and in revised form $\mathrm{xx}$ xxxx 2012)

The flow over a flat plate at a Reynolds number of 750 is numerically investigated via fine Large Eddy Simulation (LES) first, at normal $\left(90^{\circ}\right)$ and then at oblique $\left(45^{\circ}\right)$ incidence flow direction with a uniform steady inlet. The results are in complete agreement to the Direct Numerical Simulation (DNS) and experimental data, thereby serving as a validation for the present simulations. For the normal $\left(90^{\circ}\right)$ uniform inflow case, coherent vortices are alternatively shed from both leading edges of the plate, whereas for the oblique $\left(45^{0}\right)$ uniform inflow case the shedding from the two sides of the plate interact strongly resulting in a quasi-periodic force response. The normal flat plate is then analyzed with an incident gust signal with varying amplitude and time period. For these incident coherent gust cases, a reference test case with variable coherent inlet is first studied and the results are compared to a steady inlet simulation, with a detailed analysis of the flow behavior and the wake response under the incident gust. Finally, the flat plate response to 16 different gust profiles is studied. Transient drag reconstruction for these incident coherent gust cases is then presented based on frequency dependent transfer function and phase spectrum analysis.

\section{Introduction}

Two dimensional bluff bodies such as flat plates with large aspect ratios and infinite cylinders have served as geometrically simple-yet challenging test cases for many fluid dynamics research studies. For such bluff bodies, the transient three dimensional wake formation phenomenon can be analyzed to understand other complex flow configurations. The flow past a flat plate at incidence has a fixed separation point where the Reynolds $(R e)$ number only plays a role in the transition of the flow from laminar to fully turbulent; Thompson et al. (2006), Norberg (2003) and Afgan et al. (2007). Flow over such bluff bodies at high $R e$ numbers have been extensively investigated over the past; mainly related to the aerospace industry (flow over nominally two-dimensional stalled wings). For lower Re numbers, such flat plate configurations have many other practical applications. For example, flow over solar panels or road side signs/billboards, where knowing drag and other aerodynamics forces is of high importance. To provide some background, a few

$\dagger$ imran.afgan@manchester.ac.uk, Also at department of Mechanical \& Aerospace Engineering, Air University, E-9, Islamabad. 


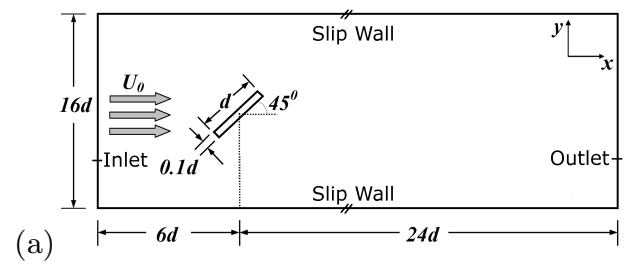

Figure 1: Flow configuration for oblique $\left(45^{0}\right)$ flat plate: (a) View in $X Y$ plane. (b) View in $X Z$ plane. (b)

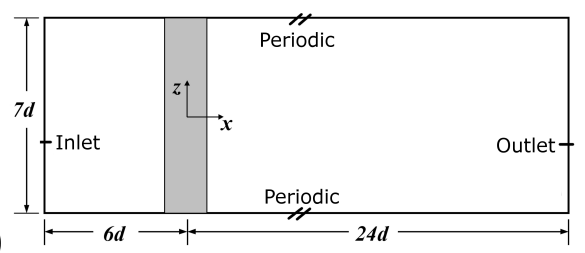

of the notable studies relating to such flows are cited here. Perhaps one of the earliest recorded studies is that conducted by Fage \& Johansen (1927) in which the authors experimentally test a three dimensional infinite flat plate with various angles of attack $\left(0-90^{0}\right)$ at a high $R e$ number of $1.5 \times 10^{5}$. The in-depth data reported by this study includes the aerodynamic force response $\left(C_{L}, C_{D}\right)$, velocity profiles along the edge of the plate and in the wake region, frequency of vortex shedding and vortex spacing. Perry \& Steiner (1987) and Steiner \& Perry (1987) present a two part experimental study for flow over oblique and normal flat plates with different aspect ratios (5, 13.2 \& 9.4) over a Re number range of 14,000 to 20,000 discussing the qualitative quasi-averaged velocity fields in the wake. Kiya \& Matsumura (1988), experimentally studied characteristics of various frequency components of the velocity fluctuations in the near wake behind a thin bevelled normal plate immersed in a uniform flow at a $R e$ number of 23,000 . The most important finding of their study was that the random shearing stress is mainly influenced by the components with frequencies of around half that of the vortex shedding frequency on the sides of the wake.

Another systematic three part notable study is that of Najjar \& Vanka (1995a), Najjar \& Vanka (1995b) and Najjar \& Balachandar (1998). In the first part the authors test a two-dimensional flat plate for Re numbers ranging from 80-1,000 using a finite difference technique. In the second part Najjar \& Vanka (1995b), a similar three-dimensional configuration is studied by Direct Numerical Simulation (DNS), with the underlying idea of correlating the aerodynamic coefficients and identifying the flow similarities between twoand three-dimensional flat plate wake formations. Najjar \& Balachandar (1998) further investigated via DNS the turbulent structure formation in the wake connected to the fluctuating drag and lift forces for a three-dimensional flat plate at a Re number of 250 . They report a low frequency unsteadiness at a period of about 10 times that of vortex shedding period, resulting in a variation of low and high drag regimes. This is attributed to the imbalance of the stream-wise and span-wise vortex formation which was not found to be in perfect synchronization.

For a flat plate with bevelled edges, Chen \& Fang (1996) experimentally study the flow over a $R e$ number range of $3.5 \times 10^{3}-3.2 \times 10^{4}$ for various angles of attack $\left(0-90^{0}\right)$. Yeung \& Parkinson (1997) present an inviscid analytical model for steady separated flow around an inclined flat plate. Their model predictions are satisfactory at only small angles of attack where classical airfoil theory is recovered. Mazharoğlu \& Hacışevki (1999) experimentally test a normal flat plate at a Re number of $3.3 \times 10^{4}$ using triple-decomposition of the instantaneous velocity into mean, coherent and turbulent parts. The decomposed velocity signals are then used directly for phase averaging thus easily highlighting the coherent structures in the wake region. Hussain (1986) discusses the characteristic measurement techniques of coherent structures in transitional and fully turbulent flows. Most of the aforementioned studies are very interesting from an academic point of point of view; presenting much insight into the flow field around bluff bodies. However, from an engi- 


\begin{tabular}{|c|c|c|c|c|c|}
\hline Study & Mode & $\mathrm{Re}$ & Geom & Angle $^{0}$ & Data Reported \\
\hline Fage \& Johansen (1927) & Exp & $1.5 \times 10^{5}$ & $\begin{array}{l}\text { Infinite 3D, } \\
\text { AR } 14\end{array}$ & 0-90 & $\begin{array}{ll}C_{L}, \quad C_{D}, & \mathrm{U} / \mathrm{U}_{0} \\
\text { Kármán } & \text { Vort. } \\
\text { Strength } & \end{array}$ \\
\hline Lamont \& Hunt (1980) & Exp & - & $\begin{array}{l}\text { Supersonic } \\
\text { jet impinge- } \\
\text { ment }\end{array}$ & $30-90$ & $\begin{array}{l}\text { Surface pressure distri- } \\
\text { butions, shock profiles }\end{array}$ \\
\hline Perry \& Steiner (1987) & $\begin{array}{l}\text { Exp/ } \\
\text { Theo }\end{array}$ & 20,000 & $\begin{array}{l}2 \mathrm{D}, 3 \mathrm{D}, \mathrm{AR} \\
5,13.2,9.4\end{array}$ & 45,90 & $\begin{array}{l}\text { St, Blockage Ratio, } \\
\bar{U}, \overline{u_{i}^{\prime} u_{i}^{\prime}} \text { contours }\end{array}$ \\
\hline Steiner \& Perry (1987) & $\begin{array}{l}\text { Exp/ } \\
\text { Theo }\end{array}$ & 20,000 & $\begin{array}{l}2 \mathrm{D}, 3 \mathrm{D}, \mathrm{AR} \\
5,13.2,9.4\end{array}$ & 45,90 & $\bar{U}, \overline{u_{i}^{\prime} u_{i}^{\prime}}$ profiles \\
\hline $\begin{array}{l}\text { Kiya } \\
(1988)\end{array} \quad \& \quad$ Matsumura & Exp & 23,000 & $\begin{array}{l}\text { Bevelled } \\
\text { 3D, AR } 15\end{array}$ & 90 & $\bar{U}, \overline{u_{i}^{\prime} u_{i}^{\prime}}$ \\
\hline Drabble et al. (1990) & Exp & $20,000-100,000$ & $3 \mathrm{D}, \mathrm{AR} 1$ & 90 & $\begin{array}{l}\text { Admittance Parame- } \\
\text { ter, } C_{L}, C_{D}\end{array}$ \\
\hline Leder (1991) & Exp & $2.8 \times 10^{4}$ & $3 \mathrm{D}$ & 90 & $\begin{array}{l}\text { St, vortex shedding } \\
\text { pattern }\end{array}$ \\
\hline Dennis et al. (1993) & $\begin{array}{l}\text { Exp/ } \\
\text { Num }\end{array}$ & 100 & $\begin{array}{l}\text { Impulsely } \\
\text { started 3D }\end{array}$ & - & $\begin{array}{l}\text { Blockage effects, wake } \\
\text { formation }\end{array}$ \\
\hline $\begin{array}{l}\text { Tamaddon-Jahromi et } \\
\text { al. (1994) }\end{array}$ & Num & 500 & $2 \mathrm{D}$ & 90 & Wake formation \\
\hline Najjar \& Vanka (1995a) & Num & 1,000 & $2 \mathrm{D}$ & 90 & $\begin{array}{l}C_{L}, C_{D}, \mathrm{U}, \mathrm{St}, \text { Block- } \\
\text { age effects }\end{array}$ \\
\hline Najjar \& Vanka (1995b) & Num & 1,000 & $3 \mathrm{D}$ & 90 & $C_{L}, C_{D}, C_{P}$ \\
\hline Chen \& Fang (1996) & Exp & $3.5 \times 10^{4}$ & $\begin{array}{l}\text { Bevelled } \\
\text { 3D, AR } 7.6\end{array}$ & $0-90$ & $\begin{array}{l}\text { St, wake formation } \\
\text { length }\end{array}$ \\
\hline $\begin{array}{l}\text { Koumoutsakos \& Shiels } \\
\text { (1996) }\end{array}$ & Num & $20-1,000$ & $2 \mathrm{D}$ & 90 & $\begin{array}{l}C_{D}, \text { Vorticity con- } \\
\text { tours, transient wake } \\
\text { streamlines }\end{array}$ \\
\hline $\begin{array}{l}\text { Yeung } \\
(1997)\end{array} \quad \& \quad$ Parkinson & Analytic & ical & - & 0 & St, $\mathrm{C}_{P}$ \\
\hline $\begin{array}{l}\text { Najjar } \& \text { Balachandar } \\
(1998)\end{array}$ & $\begin{array}{l}\text { Exp/ } \\
\text { Num }\end{array}$ & 250 & $2 \mathrm{D}, 3 \mathrm{D}$ & 90 & $C_{L}, C_{D}, \mathrm{St}$ \\
\hline $\begin{array}{l}\text { Mazharoğlu \& Hacışevki } \\
\text { (1999) }\end{array}$ & $\operatorname{Exp}$ & $3.3 \times 10^{4}$ & $3 \mathrm{D}$ & 90 & $\begin{array}{l}1^{s t}, 2^{\text {nd }} \& 3^{\text {rd }} \text { order } \\
\text { statistics }\end{array}$ \\
\hline Breuer \& Jovicic (2001) & $\begin{array}{l}\text { Num- } \\
\text { LES }\end{array}$ & 20,000 & Infinite 3D & 18 & $C_{L}, C_{D}, \mathrm{St}$ \\
\hline Julien et al. (2003) & Exp & 300 & $3 \mathrm{D}$ & - & $\begin{array}{l}\text { Wake vortex formation } \\
\text { plots }\end{array}$ \\
\hline Julien et al. (2004) & Analytic & ical & - & - & $\begin{array}{l}\text { Vortex street forma- } \\
\text { tion study }\end{array}$ \\
\hline Wu et al. (2005) & Exp & $18,000-27,000$ & $\begin{array}{l}\text { Bevelled } \\
3 \mathrm{D}, \text { AR } 4.7\end{array}$ & 90 & St, $\bar{U}, \overline{u_{i}^{\prime} u_{i}^{\prime}}$ \\
\hline Saha (2007) & DNS & 150 & - & - & $\begin{array}{l}\text { PDF, FFT, vorticity } \\
\text { plots }\end{array}$ \\
\hline $\begin{array}{l}\text { Narasimhamurthy \& An- } \\
\text { dersson (2009) }\end{array}$ & DNS & 750 & Infinite 3D & 90 & $\begin{array}{l}C_{P}, 1^{s t}, 2^{\text {nd }} \& 3^{r d} \text { or- } \\
\text { der statistics }\end{array}$ \\
\hline Current Study & $\begin{array}{l}\text { Fine } \\
\text { LES }\end{array}$ & 750 & $\begin{array}{l}\text { Infinite 3D, } \\
\text { Variable in- } \\
\text { lets }\end{array}$ & 90,45 & $\begin{array}{l}C_{P}, 1^{s t}, 2^{n d} \& 3^{\text {rd }} \\
\text { order statistics, ad- } \\
\text { mittance parameters, } \\
\text { drag reconstruction }\end{array}$ \\
\hline
\end{tabular}

Table 1: Summary of notable experimental, numerical and analytical studies conducted to date for flow over a Flat Plate. 
neering perspective, for many practical applications, the incoming flow is neither uniform nor normal to the bluff body. Thus, one cannot directly correlate the flow behavior to arbitrarily inclined bluff bodies with non-uniform inlet conditions, which have a far more complicated wake behavior.

For unsteady flows, Koumoutsakos \& Shiels (1996) simulated a normal impulsively started flat plate giving an in-depth description of the transient wake development and the aerodynamic coefficients for a fairly low $R e$ number. However, no attempt was made in this study to look into the wake behavior with an oscillatory inlet and/or model. Drabble et al. (1990) present a unique scenario relating the stream-wise fluctuating forces on the flat plate with the inlet flow velocity fluctuations through a frequency dependent transfer function called the Admittance Parameter. Even though the admittance function is strongly dependent upon the geometric shape of the configuration, its response in the two incoming flow cases (turbulent and coherent), is reported to be quite the opposite. The present study tries to mimic this behavior by introducing coherent yet varying gust signals: a series of coherent incident gusts superimposed on the mean inlet velocity. The response of the plate in terms of aerodynamic coefficients and aerodynamic admittance is then studied to understand the physics of the flow. A similar experimental study conducted on actual roadside signs by Quinn, Baker \& Wright (2001) reports the aerodynamic forces and the wind admittance parameters. However, the authors do not discuss the underlying flow physics, let alone the profile of the inlet conditions or the plate response to it.

More recently Narasimhamurthy \& Andersson (2009), performed a Direct Numerical Simulation of flow over a normal flat plat at a Re number of 750 based on the plate width. The study concisely reports the Strouhal number, velocity profiles and Reynolds stresses in the wake region. For the uniform inlet cases of the current study $\left(90^{0}\right.$ normal flat plate) comparisons are based on the data of the aforementioned DNS of Narasimhamurthy \& Andersson (2009). Other notable studies of similar configurations are those of Julien, Lasheras \& Chomaz (2003), Julien, Ortiz \& Chomaz (2004), Wu et al. (2005), Saha (2007), Kim et al. (2004) and Nakagawa et al. (1999). A detailed table showing the tested configurations and summary of available results are presented in Table 1.

The chosen test cases under uniform and unsteady inlet conditions are very interesting from an engineering perspective; the stabilizing fins and rudders of sub-marines/ships (which experience varying angles of attach), down-force spoilers of race cars (naturally set at high oblique angles, $10-35^{0}$ ), mixer grids in nuclear fuel rod bundles and wind turbines. Such geometrically similar configurations rarely experience an upstream uniform flow. In fact, the inflow conditions are strongly varying; for example, the flapping wings of insects (which can be generalized as finite flat plates with sinusoidal or varying coherent inlets). Thus, from a practical point of view, it is very interesting, to not only study the flow over arbitrarily inclined configurations, but also to use the obtained knowledge to look at similar geometries with varying inflow conditions. Then, looking into a methodology for the response prediction of such complex cases would be a very novel contribution to the field of fluid mechanics.

It is thus envisioned that the current paper will present a better understanding of flow over bluff bodies and their response to varying inlet conditions. The paper starts with the test case description and discussion of numerical treatment for simulations (Secs. 2 \& 3), followed by the discussion of results for uniform inlet cases (Sec. 4). Results for incident coherent cases are then presented in Sec. 5. Finally, the fluctuating response and drag reconstruction are reported in Sec. 6. 
(a)

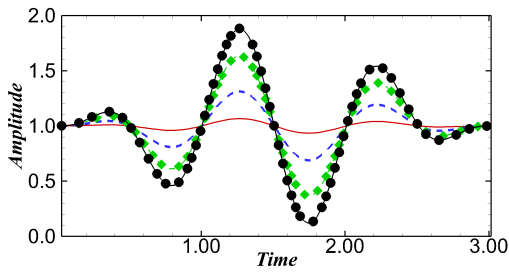

(b)

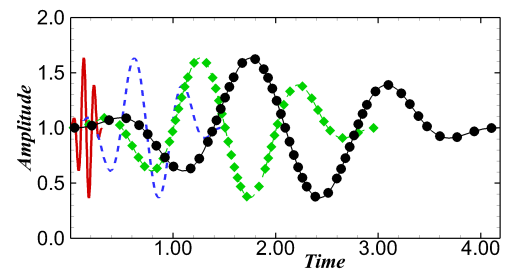

Figure 2: Gust profile for coherent inlet cases with varying gust amplitude $(A)$ and time cycle $(T)$ : (a) Different amplitudes $(-A 1,--A 2,-\wedge 3,-\mathbf{-}-A 4)$ with constant time period $(T=3.01 \mathrm{~s})$. (b) Constant maximum amplitude $(A=0.67)$ with different time periods (-T1,- - T2, - - T3, - - $T 4)$.

\section{Case Description}

For the uniform inlet flow, two configurations have been numerically investigated; Case 1: Normal flat plate with inflow angle of $90^{\circ} \&$ Case 2: Oblique plate at $45^{0}$ to the inflow direction. The three-dimensionality of the wake formation for bluff bodies is attributed to the flow intrinsic and extrinsic effects as discussed in detail by Roshko (1993). A key parameter dictating the extrinsic effect is the aspect ratio of the bluff body as reported by Fox \& West (1990) and Norberg (1994). Keeping this under consideration, a spanwise correlation of the stream-wise $(u)$ and cross-stream $(v)$ velocities (not shown in this article) led to the conclusion that an $A R$ of 7 was sufficient for the current configuration. The chord of the plate was kept at $1 d$ and the thickness $0.1 d$ (see figure 1 for complete geometric details and boundary conditions).

Determination of an accurate gust model is a dynamic research topic; many stochastic and probabilistic approaches have been adopted in the past to present the gust behavior, see Goyette, Brasseur \& Beniston (2003), Bierbooms \& Cheng (2002) and Bierbooms (2004). In the latter two, the authors present a stochastic approach to model gust for wind turbine designs. The gust excited response of structures is discussed in detail and a probabilistic 3-D turbulence model for gust buffeting is presented by Solari \& Piccardo (2000), the gust buffeting and turbulence uncertainties in atmospheric flows is then discussed later by Pagnini \& Solari (2002). In the current study, a realistic fit of the gust profile of Bierbooms \& Cheng (2002) and Bierbooms (2004) is chosen as given below.

$$
u(t)=U_{0}+A \frac{t^{2}(t-T)^{2}}{(T / 2)^{4}} \sin \left(\frac{2 \pi}{T}(3 t+T)\right), t \in[0, T]
$$

where $A \in[0,1]$ is the maximum gust amplitude (yielding a maximum $U_{\text {gust }}$ of $2 U_{0}$ ) and $T \in[0,4.5]$ is the duration (time period) of the gust. This gust period is set to match the wind averaging periods. Generally, for a 10 minute averaging period, a gust between 1 and 5 seconds is recommended, see Harper, Kepert \& Ginger (2008) for more details. To discretize the gust model in two dimensions ( $A$ and $T$ ), a n-point Gauss-Legendre quadrature formula given by Press et al. (1996) is utilized.

Each incident gust cycle for the coherent inlet simulations is resolved over a fixed number of time steps; 1024. This leads to a strong dependence of the non-dimensional numerical time step $\left(\Delta t^{+}=\Delta t U_{0} / d\right)$, upon the time period of the gust oscillation $(T)$. However, this ensures that each gust profile is adequately resolved, especially for the cases where the amplitude $(A)$ is high and the time $\operatorname{period}(T)$ is very small. For the coherent inlet gust simulations, a reference gust test case is chosen, which is later used for comparison of mean statistics and instantaneous quantities with the uniform inlet 


\begin{tabular}{cccc}
\hline & $\begin{array}{c}\text { Normal }\left(\mathbf{9 0}^{0}\right) \\
\text { uniform inlet }\end{array}$ & $\begin{array}{c}\text { Oblique }\left(\mathbf{4 5}^{0}\right) \\
\text { uniform inlet }\end{array}$ & $\begin{array}{c}\text { Normal }\left(\mathbf{9 0}^{0}\right) \\
\text { coherent inlet }\end{array}$ \\
Coarse Mesh(CM) & $7.6 / 96 N_{Z}, Y^{+} \approx 2$ & $8.2 / 96 N_{Z}, Y^{+} \approx 2$ & - \\
Medium Mesh(MM) & $11.64 / 128 N_{Z}, Y^{+}<1$ & $12.14 / 128 N_{Z}, Y^{+}<1$ & $11.64 / 128 N_{Z}, Y^{+}<1$ \\
Fine Mesh(FM) & $16.2 / 120 N_{Z} Y^{+}<1$ & $17.3 / 120 N_{Z}, Y^{+}<1$ & - \\
Very Fine Mesh(VFM) & $19.8 / 180 N_{Z}, Y^{+}<1$ & - & -
\end{tabular}

Table 2: Overview of the grid sensitivity study: cases simulated, meshes tested in million of cells/the total number of cells in the span-wise $\left(N_{Z}\right)$ direction, maximum value of $Y^{+}$.

(a)

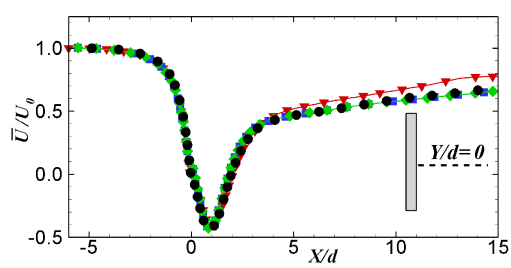

(b)

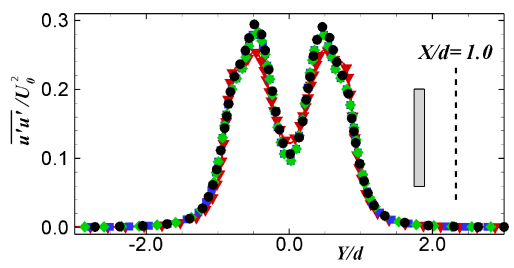

Figure 3: Comparisons of $-\mathbf{\nabla}-C M$, $M M \&-F M$ against $D N S$ of Narasimhamurthy \& Andersson (2009) for normal flat plate with uniform inlet: (a) Mean $\left(\bar{U} / U_{0}\right)$ profile along symmetry line $Y / d=0.0$. (b) Stream-wise Reynolds normal stress $\left(\overline{u^{\prime} u^{\prime}} / U_{0}\right)$ profile along $X / d=1.0$.

case. Some characteristic gust profiles over the range of the chosen quadrature points in real space are shown in figure 2 with varying amplitude and time periods. A total of 16 Large Eddy Simulation (LES) runs were performed for different combinations of $A$ and $T$ where the time dependent inlet boundary condition becomes a repetition of the same signal, thus leading to incident coherent gusts.

\section{Numerical Treatment}

An incompressible collocated finite volume code for complex geometries, Code_Saturne (Archambeau et al. 2004), is used to solve 3D Navier-Stokes equations on unstructured grids. For LES, the standard Smagorinsky model with Van Driest wall damping function is used. A $2^{\text {nd }}$ order pure central differencing scheme is utilized for spatial discretization whereas the pressure-velocity coupling is ensured by the SIMPLEC algorithm; Van Doormal \& Raithby (1984), with Rhie and Chow interpolation (see Rhie \& Chow (1982)) in the correction step to avoid oscillatory solutions. For temporal discretization, Crank-Nicolson with linearized convection is used. For further details about the numerical treatment and Code_Saturne see Benhamadouche et al. (2005), Moulinec et al. (2005), Afgan et al. (2007).

To avoid needless refinement in both far wake and far normal locations an O-grid was generated around the flat plate which was encapsulated inside a rectangular domain. The 2-dimensional mesh was then extruded in the span-wise direction with uniform cell spacing. Such grid techniques have been suitably tested in the past, see Afgan et al. (2007). Three different meshes were tested; a coarse mesh $(C M)$ with 7.6 million cells, a medium mesh $(M M)$ with 11.6 million cells and a fine mesh $(F M)$ with 16.2 million cells. The details of these meshes are given in table 2 along with maximum $Y^{+}$values and 
the number of cells in the span-wise direction. The $Y^{+}$distributions along the leeward surface and the side edge of flat plate are always less than one for $M M, F M$ and $V F M$. Figure 3 shows both mean velocity and the Reynolds stream-wise normal stress for all grids $(C M, M M \& F M)$ for the normal $\left(90^{\circ}\right)$ case with uniform inlet. It can be observed that no marked improvement in results is achieved by further refining the grid beyond $M M$ resolution, especially in the plate wake region which is of interest in the current study. Hence, the $M M$ was taken as the benchmark resolution for all further simulations (both uniform and coherent inlet cases).

In addition to the grid sensitivity study, away from the body, a spatial (grid) resolution estimation was also conducted by comparing larger flow length scales $\left(L=k^{3 / 2} / \varepsilon\right)$, the Taylor length scales $\left(\lambda=(10 \nu k / \varepsilon)^{1 / 2}\right)$, the Kolmogorov scales $\left(\eta=\left(\nu^{3} / \varepsilon\right)^{1 / 4}\right)$ and the space filter size $\left(\Delta=\operatorname{Vol}^{1 / 3}\right)$ of the three meshes tested $(C M, M M \& F M)$. Here, $k$ is the resolved turbulent kinetic energy computed as $k=\left(\overline{u^{\prime 2}}+\overline{v^{\prime 2}}+\overline{w^{\prime 2}}\right) / 2$ and $\varepsilon$ is the dissipation rate of kinetic energy defined as $\varepsilon=\varepsilon_{V i s}+\varepsilon_{S G S}$, where $\varepsilon_{V i s} \approx$ $\nu\left(\overline{\left(\partial u_{i}^{\prime} / \partial x_{j}\right)\left(\partial u_{i}^{\prime} / \partial x_{j}\right)}\right.$ and $\varepsilon_{S G S}=\nu_{t}\left(\overline{\left(\partial u_{i}^{\prime} / \partial x_{j}\right)\left(\partial u_{i}^{\prime} / \partial x_{j}\right)}\right.$. Both $k$ and $\varepsilon$ have been computed for the medium mesh $M M$ simulation. It is observed from figures $4 \mathrm{a}$ and $\mathrm{b}$ that the $M M$ is almost of the same resolution as that of Kolmogorov scales both on the side of the plate and in its wake along the tip region. The present $M M$ is considerably fine even in the span-wise direction when compared to the DNS studies of Yao et al. (2001), Moser \& Blachandar (1998) and Narasimhamurthy \& Andersson (2009). A similar estimation of the numerical time step is based on a comparison between integral time scales $\left(L_{\tau}=k / \varepsilon\right)$, the Taylor time scales $\left(\lambda_{\tau}=(15 \nu / \varepsilon)^{1 / 2}\right)$ and the Kolmogorov time scales $\left(\tau=(\nu / \varepsilon)^{1 / 2}\right)$, see figure 4c. The effects of the sub-grid Scale (SGS) contribution and dynamic model were also checked but the results are not presented in the current paper. The readers are encouraged to see Afgan et al. (2011) for a detailed investigation of SGS contribution with a similar numerical treatment. The simulations were performed using a total of 2048 processors for each job on Électricité de France (EDF) Blue Gene/P supercomputer for $150 \mathrm{CPU}$ (central processing unit) hours i.e. around 12 through-flow passes $\left(\operatorname{Pass}_{F} T=\right.$ $\left.N \Delta t U_{0} / L_{X}\right)$. For all uniform inlet cases, the flow was allowed to develop for $4\left(\operatorname{Pass}_{F} T\right)$ and averaged over the last $8\left(\operatorname{Pass}_{F} T\right)$. The total time averaging period of the statistics translates into approximately 240 large eddy turnover time $\left(t_{e}=D / U_{0}\right)$. At the end of the simulations the results were also space averaged in the homogeneous $(Z)$ direction. The simulations with incident coherent gusts were restarted from the uniform flow field and were run for at least 60 complete gust cycles which corresponds to about 6-9 throughflow passes.

\section{Results and Discussion for Uniform Inlet Cases}

\subsection{Mean Pressure Distribution and Aerodynamic Forces}

The mean pressure coefficients $\left(\bar{C}_{P}=2\left(\bar{P}-P_{r e f}\right) / \rho U_{0}^{2}\right)$ for both normal $\left(90^{0}\right)$ and oblique $\left(45^{0}\right)$ cases (LES $\left.M M\right)$ with uniform inlet are shown in figure 5 against the measurements of Fage \& Johansen (1927), DNS of Najjar \& Vanka (1995b) at Re 1000, Najjar \& Balachandar (1998) at Re 250 and Narasimhamurthy \& Andersson (2009) at $R e$ 750. On the upstream surface (front-windward side) the current LES and DNS of Narasimhamurthy \& Andersson (2009) compare well, whereas the DNS of Najjar \& Vanka (1995b) has a slightly wider mean pressure distribution especially near the edges of the flat plate. On the downstream surface (back-leeward side) once again the current LES and DNS of Narasimhamurthy \& Andersson (2009) compare well, whereas the DNS of Najjar \& Vanka (1995b) under predicts the mean pressure distribution, which is complemented 
(a)

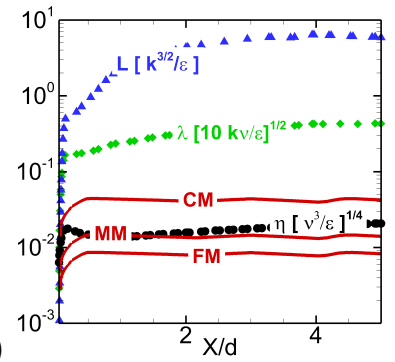

(b)

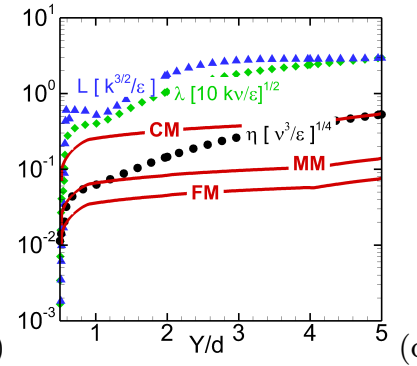

(c)

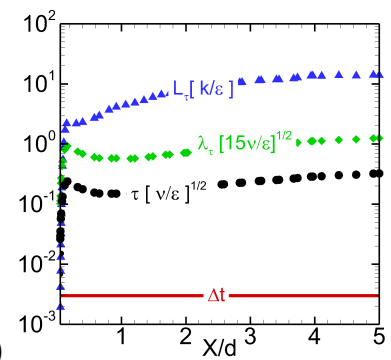

Figure 4: Comparisons between larger flow scales $\left(-\boldsymbol{\Delta}-L=k^{3 / 2} / \varepsilon, L_{\tau}=k / \varepsilon\right)$, the Taylor scales $\left(-\downarrow-\lambda=(10 \nu k / \varepsilon)^{1 / 2}, \lambda_{\tau}=(15 \nu / \varepsilon)^{1 / 2}\right)$, the Kolmogorov scales $\left(\bullet \eta=\left(\nu^{3} / \varepsilon\right)^{1 / 4}\right.$, $\left.\tau=(\nu / \varepsilon)^{1 / 2}\right)$ and the grid scales $(\Delta \& \Delta t)$ for the three meshes tested $(C M, M M \&$ $F M)$. (a) Length scale comparison in the wake of the plate at $Y / d=0.5$ along $X$-axis. (b) Length scale comparison on the side of the plate at $X / d=0$ along $Y$-axis. (c) Time scale comparison.

$\begin{array}{lccccccc} & R e & S t=f d / U_{0} & \bar{C}_{D} & C_{D}^{\prime} & \bar{C}_{L} & C_{L}^{\prime} & L_{R} / d \\ \text { Present LES 90 } & 750 & 0.167 & 2.29 & 0.251 & 6 \times 10^{-5} & 0.008 & 1.88 \\ \text { Narasimhamurthy \& Andersson } & 750 & 0.168 & 2.31 & - & - & - & 1.96 \\ \text { (2009) 90 } & & & & & & & \\ \text { Najjar \& Vanka (1995b) } 90^{0} & 1000 & 0.143 & 2.26 & - & - & - & 2.55 \\ \text { Najjar \& Balachandar (1998) } & 250 & 0.161 & 2.36 & - & - & - & 2.35 \\ 90^{0} & & & & & & & \\ \text { Fage \& Johansen (1927) } 90^{0} & 1.5 \times 10^{5} & 0.146 & 2.13 & - & - & - & - \\ \text { Fage \& Johansen (1927) } 45^{0} & 1.5 \times 10^{5} & 0.205 & 1.80 & - & - & - & - \\ \text { Present LES 45 } & 750 & 0.206 & 1.95 & 0.328 & 1.83 & 0.562 & 0.62\end{array}$

Table 3: Comparisons between Strouhal number $(S t)$, mean drag \& lift coefficients $\left(\bar{C}_{D}\right.$, $\left.\bar{C}_{L}\right)$, rms of drag \& lift coefficients $\left(C_{D}^{\prime}, C_{L}^{\prime}\right)$ and mean recirculation length $\left(L_{R} / d\right)$.

by an over prediction of the mean recirculation length, essentially leading to a lower Strouhal number (see Table 3). Narasimhamurthy \& Andersson (2009) attribute this discrepancy of higher pressure recovery in DNS data of Najjar \& Vanka (1995b), to a numerical artifact due to low grid resolution. For the oblique $\left(45^{\circ}\right)$ flat plate configuration the LES predictions on the pressure side compare well to the measurements of Fage \& Johansen (1927) (49.85 ${ }^{\circ}$ flat plate), see figure 5b. However, on the back side current LES shows a varying pressure change with a substantially lower plateau of around $\bar{C}_{P}=-2.2$. This difference in results is attributed to the very high $R e$ number used by Fage \& Johansen (1927). It is interesting to note that the difference in mean pressure coefficient between the front and back surfaces (defined as $\Delta \bar{C}_{P}=\left(\bar{C}_{P}\right)_{\text {front }}-\left(\bar{C}_{P}\right)_{\text {back }}$ ) remains almost unchanged along the length of the flat plate, as shown by the vertical lines $L 1$, L2 and L3 in figure $5 \mathrm{~b}$.

The instantaneous lift $\left(\left(C_{L}=2 F_{L} / \rho U_{0}^{2} A\right)\right)$ and $\operatorname{drag}\left(\left(C_{D}=2 F_{D} / \rho U_{0}^{2} A\right)\right.$ coefficients and their spectrum for both the cases are shown in figure 6 , where $A$ is the projected normal area. For the normal $\left(90^{0}\right)$ flat plate, a Strouhal number $\left(S t=f d / U_{0}\right)$ of 0.167 is found, which is in excellent comparison with the DNS of Narasimhamurthy \& Andersson (2009) $(S t=0.168)$, see table 3 . The mean lift $\left(\bar{C}_{L}\right)$ is close to zero showing that the first 3 digits of the other statistical results are meaningful. For the oblique $\left(45^{0}\right)$ flat plate, the 
(a)

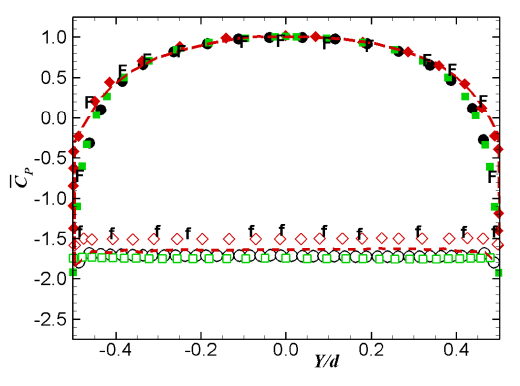

(b)

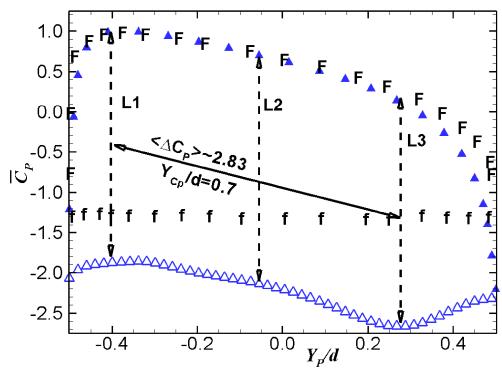

Figure 5: Mean $C_{P}$ distribution on the surface of the plate; DNS ( $\diamond$ front, $\diamond$ back) Najjar \& Vanka (1995b), DNS ( front, $\bigcirc$ back) Narasimhamurthy \& Andersson (2009), DNS (- front, -- back) Najjar \& Balachandar (1998), experimental (-F- front, -f- back) Fage \& Johansen (1927), LES normal plate with uniform inlet $M M$ ( $\mathbf{\square}$ front, $\square$ back), LES oblique plate with uniform inlet $M M$ ( $\boldsymbol{\Delta}$ front, $\triangle$ back): (a) Normal $\left(90^{\circ}\right)$ flat plate. (b) Oblique $\left(45^{0}\right)$ flat plate.

(a)

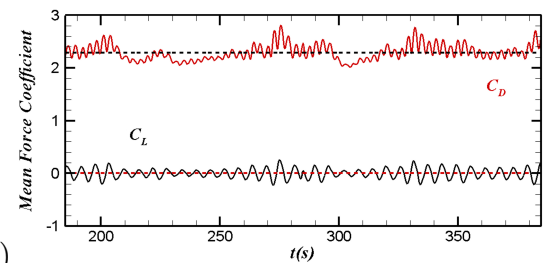

(c)

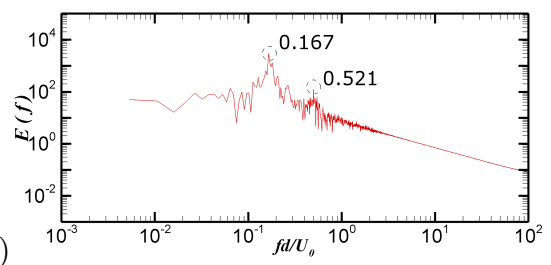

(b)

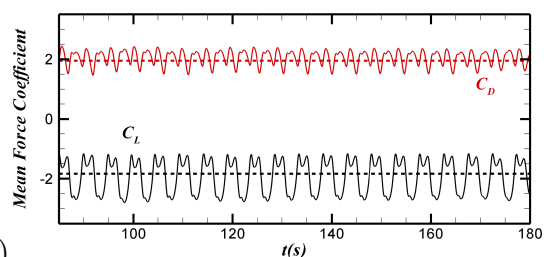

(d)

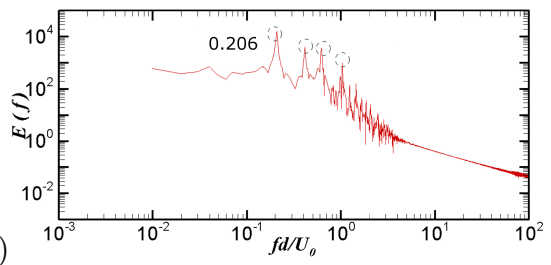

Figure 6: (a) Instantaneous lift $\left(C_{L}\right.$-lower curve $)$ and drag $\left(C_{D}\right.$-upper curve) coefficients for normal $90^{\circ}$ flat plate. (b) Instantaneous lift $\left(C_{L}\right.$-lower curve) and $\operatorname{drag}\left(C_{D^{-}}\right.$-upper curve) coefficients for oblique $45^{0}$ flat plate. (c) Power spectrum of $\left(C_{L}\right)$ for normal $90^{0}$ flat plate. (d) Power spectrum of $\left(C_{L}\right)$ for oblique $45^{0}$ flat plate.

mean lift $\left(\bar{C}_{L}\right)$ and drag $\left(\bar{C}_{D}\right)$ coefficients are -1.83 and 1.95 , respectively. Observing figure $6 \mathrm{~b}$, one notices a quasi-periodic force response for both the $C_{L}$ and $C_{D}$ profiles, this is attributed to the out-of-phase vortex pair shedding where the top and bottom vortices are shed at different frequencies leading to this multiple frequency/quasi-periodic force response. The lift force spectrum in figure $6 \mathrm{~d}$, shows four pronounced shedding frequencies, the most energetic one corresponding to the principal vortex frequency with $S t=0.206$.

\subsection{Mean Velocity Profiles and Wake Vortex Evolution}

The mean stream-wise and cross-stream velocity profiles along the wake centerline for normal $\left(90^{\circ}\right)$ and oblique $\left(45^{\circ}\right)$ cases with a uniform inlet are shown in figures $7 \mathrm{a} \&$ $\mathrm{b}$, respectively. In figure $7 \mathrm{a}$, for the normal $\left(90^{\circ}\right)$ case, the mean stream-wise velocity $\left(\bar{U}_{90} / U_{0}\right)$ has a maximum deficit of $0.44 U_{0}$ and the mean recirculation length is $L_{R} / d=$ 1.88. For the oblique $\left(45^{0}\right)$ case, this deficit is only $0.25 U_{0}$, about $50 \%$ less than the 
(a)
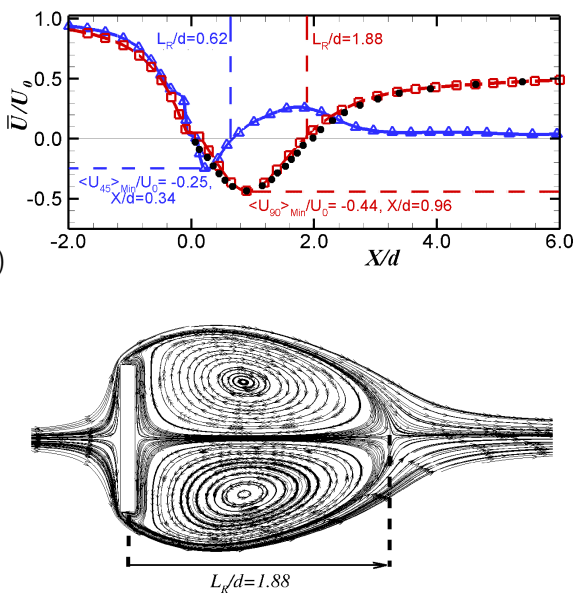

(c)

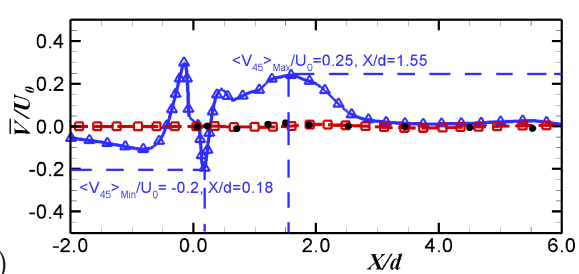

(b)

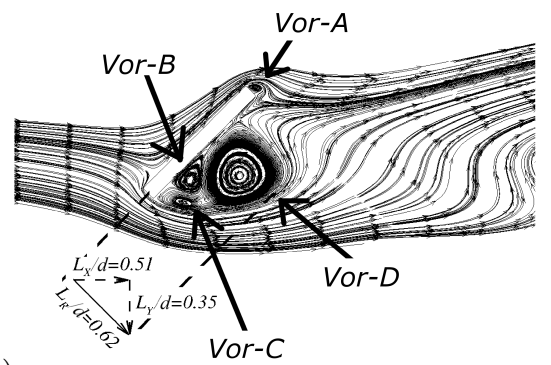

(d)

Figure 7: • Narasimhamurthy \& Andersson (2009), $\square$ LES Normal $\left(90^{\circ}\right)$ case, $\triangle$ LES oblique $\left(45^{0}\right)$ case: (a) Mean profile for stream-wise velocity $\left(\bar{U} / U_{0}\right)$. (b) Mean profile for cross-stream velocity $\left(\bar{V} / U_{0}\right)$. (c) Mean streamlines, LES Normal $\left(90^{\circ}\right)$ case. (d) Mean streamlines, LES oblique $\left(45^{0}\right)$ case.

normal $\left(90^{\circ}\right)$ case. The mean recirculation length is $L_{R} / d=0.62$ as seen from the mean stream-wise velocity $\left(\bar{U} / U_{0}\right)$ in figure $7 \mathrm{a}$. On the other hand, the mean cross-stream velocity profile for the oblique $\left(45^{0}\right)$ case shows a complex flow behavior along the wake centerline (see figure $7 \mathrm{~b}$ ). The sharp dip in mean cross-stream velocity and then a double peak higher plateau is an artifact due to plotting the velocity along the wake centerline $(X$-axis) as it is cutting through the main bigger vortex $\operatorname{Vor}-D$ (see figure $7 \mathrm{~d}$ ).

For the normal $\left(90^{\circ}\right)$ configuration, the flow separates at both the lower- and the upper-leading edges of the plate, as expected from flow past any symmetric bluff body. However, for the oblique $\left(45^{0}\right)$ configuration, the flow exhibits a local separation at the top leading edge followed by a reattachment close to the trailing edge (see the streamlines of velocity around the $V$ or $-A$ in figure $7 \mathrm{~d}$ ); this is perhaps due to the $10 \%$ thickness of the plate. After toppling over, the flow streamlines remain attached to the hind-side (back surface) of the plate until $Y / d=0.25$, where it is shed downstream. On the other hand, at the lower end of the plate, the separation point is still located at the sharp leading edge, the origin point of the $V o r-C$. This leads to the formation of several mean vortices in the plate wake, as indicated earlier and shown in the figure $7 \mathrm{~d}$. The streamline patterns are similar to the experimental results of Perry \& Steiner (1987) for the same configuration but at a much higher Re number. The authors reported that clearly there was no antisymmetric vortex shedding unlike the flow over a normal plate. In fact, the phase integrated streamline pattern showed the existence of multiple saddle points and vortices in the wake, close to the current findings. However, they reported that the temporal mean velocity plots showed only a single mean vortex bubble at one end of the plate which was mainly attributed to the longer period that it stayed in the wake than the shedding from the other end.

The temporal evolution of these four wake vortices (Vor $-A, \operatorname{Vor}-B$, Vor $-C$ $\& V o r-D)$ can better be explained with the help of vorticity plots of figure 8 . It is noted from figure $8 \mathrm{a} \& \mathrm{~h}$, that the leading bottom edge of the plate gives rise to a main 
vortex (Vor $-C$, rotating counter-clockwise), which is then stretched along the leeward side of the plate into an ellipsoidal shape giving rise to a secondary vortex (Vor $-B$ rotating clockwise, see figures $8 \mathrm{c}, \mathrm{d} \& \mathrm{e}$ ). Meanwhile, at the top end of the plate there is a strong shear layer separation near the leading edge and then reattachment near the trailing edge, causing shedding of main vortex $\operatorname{Vor}-D$ (rotating clockwise, see figures $8 \mathrm{e} \& \mathrm{f}$ ). This $V$ or $-D$ structure also triggers generation of a secondary vortex ( $V o r-A$ rotating counter-clockwise, see figures $8 \mathrm{~g} \& \mathrm{~h}$ ). However, Vor $-A$ is much short lived as it is stretched and annihilated by the toppling over flow. What is interesting to note from the whole shedding cycle is that the two main vortices, $\operatorname{Vor}-C$ and $\operatorname{Vor}-D$, shed from the opposite leading edges of the plate (bottom and top, respectively), each gives rise to a counter rotating secondary vortex which is fairly short lived. However, both these secondary vortices are generated near the top trailing edge of the plate, which essentially means that the top end of the plate generates three vortex sheddings, where as the bottom end experiences only one vortex shedding. This is thought to be the main reason behind the quasi-periodic force response of the plate as a whole. The presence of a strong shear layer leads to the generation of these secondary and additional smaller Kelvin-Helmholtz vortices, but these are short lived and of much higher frequency than the Kármán vortices. In fact, looking at the spectrum in figure $6 \mathrm{~d}$, one observes higher frequency content, which is not seen in the normal $\left(90^{\circ}\right)$ case (figure $6 \mathrm{c}$ ).

The mean stream-wise $\left(\bar{U} / U_{0}\right)$ and cross-stream $\left(\bar{V} / U_{0}\right)$ velocity profiles at various downstream locations in the wake of the plate for both $90^{\circ}$ and $45^{\circ}$ incidences are shown in figure 9. For the $90^{\circ}$ case, the maximum velocity deficit is close to $0.44 U_{0}$ which is in good agreement to the DNS of Narasimhamurthy \& Andersson (2009). Moving downstream, this deficit has now almost fully recovered at around $X / d=2.0$, where the mean stream-wise $\left(\bar{U} / U_{0}\right)$ is close to zero. Thus, the location (also the end of the recirculation region) is referred to as the free stagnation point in the past (see Fage \& Johansen (1927) and Narasimhamurthy \& Andersson (2009)). It is further noted from the same figure $9 \mathrm{a}$ that the maximum stream-wise $\left(\bar{U} / U_{0} \approx 1.35\right)$ velocity is located at around $Y / d \approx 1.0$ on the side of the plate along the line $X / d=1.0$. After $X / d=1.0$, a gradual decrease in the mean stream-wise $\left(\bar{U} / U_{0}\right)$ velocity outside of the recirculation region and a deficit recovery inside the wake recirculation takes place. Coming to the mean cross-stream $\left(\bar{V} / U_{0}\right)$ velocity profiles in figure $9 \mathrm{~b}$ for the same normal $\left(90^{0}\right)$ case, one notices the existence of the largest cross-stream velocity magnitude of $\bar{V} / U_{0}=0.2$, which is again nearly located at the free stagnation point (along line $X / d=2.0$ ). Moving downstream, one notices that the cross-stream velocity recovers back to zero at a much faster rate than the stream-wise velocity (see figures $9 \mathrm{a} \& \mathrm{~b}$ ).

From the streamline pattern of figure $7 \mathrm{c}$ and the velocity profiles shown in figure $9 \mathrm{~b}$, one notices that outside of the recirculation region, the cross-stream $\left(\bar{V} / U_{0}\right)$ velocity is directed away from the symmetry line (see the direction of $\bar{V} / U_{0}$ along $X / d=1.0$ line before $\mathrm{Y} / \mathrm{d}=-1.85)$. However, inside and downstream of the recirculation region, the velocity is directed towards the symmetry line (notice the flow normality after the $X / d=2.0$ line in figure $9 \mathrm{~b})$. For the oblique $\left(45^{0}\right)$ case, one observes a similar behavior of $\bar{V} / U_{0}$ at or before $X / d=1.0$ line, where the flow is directed away from the symmetry line. However, moving downstream, this behavior continues till $X / d=7.5$ before the flow actually starts to be directed towards the symmetry plane (see the continued opposite response of $\bar{V} / U_{0}$ profiles along $X / d=2,3,4 \& 5$ lines). This can also be judged by the streamlines of velocity behind the oblique $\left(45^{0}\right)$ plate shown in figure $7 \mathrm{~d}$. For the uniform inlet cases, the mean Reynolds stress profiles, fluctuating kinetic energy $\left(k / U_{0}^{2}\right)$ and the decay of its dissipation rate $\left(\varepsilon \nu / U_{0}^{4}\right)$ at various downstream locations were also compared to DNS of Narasimhamurthy \& Andersson (2009) which is shown in the appendix A. 

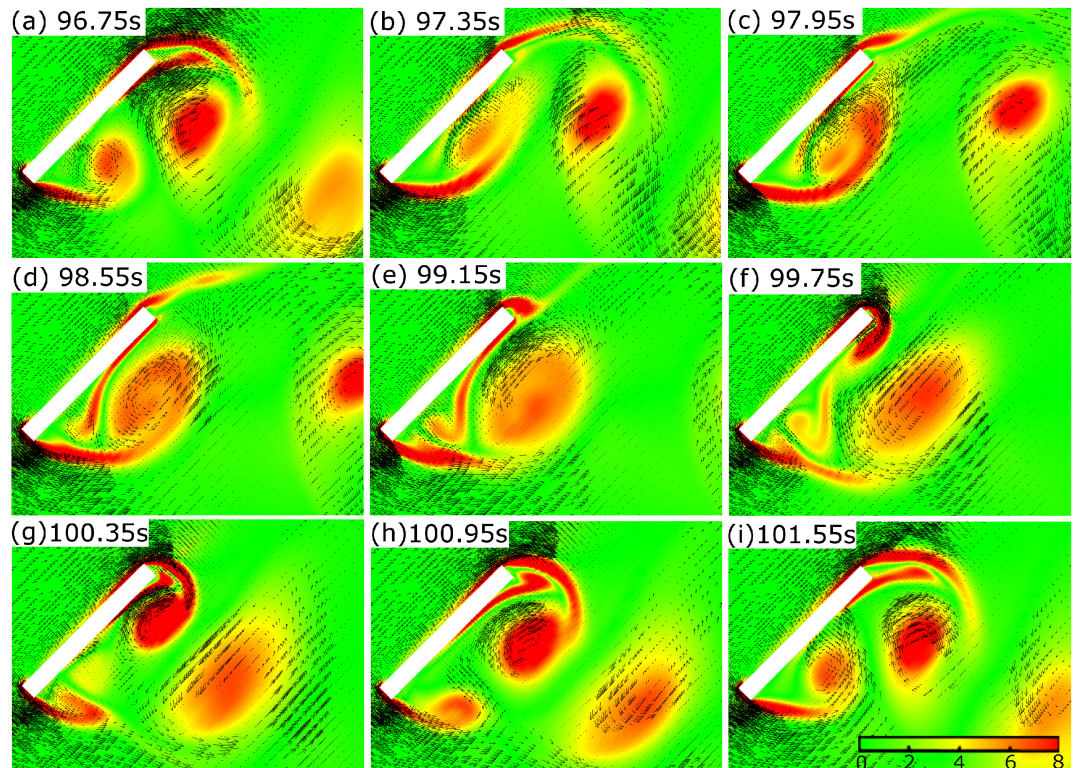

Figure 8: Near wake vortex evolution; contours of vorticity superimposed by velocity vectors at different time instances.

(a)
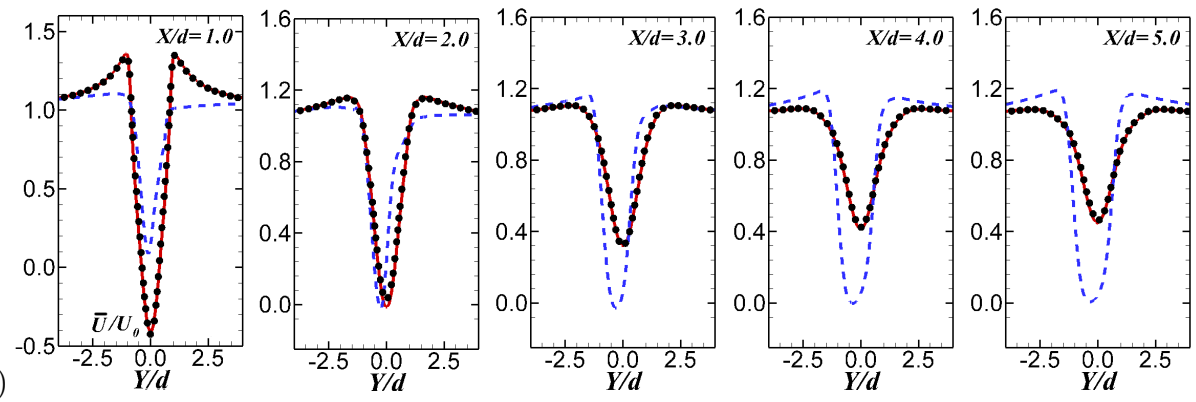

(b)
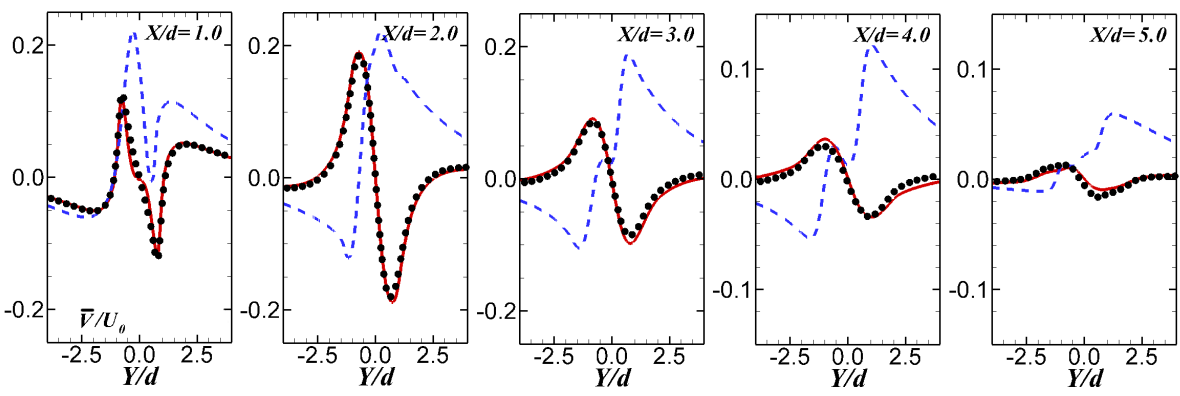

Figure 9: Mean velocity profiles along the height of the plate ( Narasimhamurthy \& Andersson (2009), - LES normal $\left(90^{\circ}\right)$ and - - LES oblique $\left.\left(45^{0}\right)\right)$ at various $X / d$ downstream locations: (a) Stream-wise velocity $\left(\bar{U} / U_{0}\right)$. (b) Cross-stream velocity $\left(\bar{V} / U_{0}\right)$. 
(a)

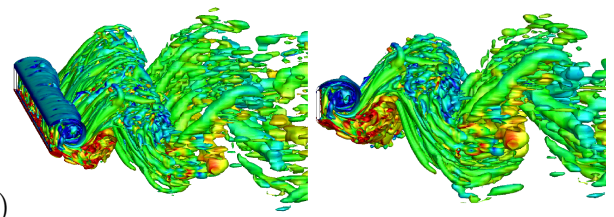

(b)

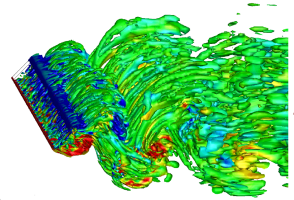

Figure 10: Isometric and side views of the IsoQ $\left(\mathrm{Q}=-5\left(S_{i j} S_{i j}-\Omega_{i j} \Omega_{i j}\right)\right.$ (a) Normal $\left(90^{0}\right)$ case (b) Oblique $\left(45^{0}\right)$ case.

In order to understand the wake formation behind the flat plates for both the normal $\left(90^{0}\right)$ and oblique $\left(45^{0}\right)$ configurations, instantaneous realizations of $(Q)$, the $2^{\text {nd }}$ invariant of the velocity gradient tensor were calculated as shown in figures 10. For computations, $Q$ is defined as $Q=-0.5\left(S_{i j} S_{i j}-\Omega_{i j} \Omega_{i j}\right)$ (see Hunt, Wray \& Moin (1988)), herein, normalized by $\left(Q=\alpha\left(U_{0} / d\right)^{2}\right)$ where $\alpha$ is a non-dimensional constant set by the user in order to obtain desired flow structures (for the current cases $\alpha$ is chosen to return a value of $\left.Q=1 / s^{2}\right)$. For the normal $\left(90^{0}\right)$ case, it is observed that the shear layer extrusion downstream before roll-up is around $1.5 d$ and the shape of this top coherent structure is rather cylindrical, as can be seen from the figure 10a. On the other hand, the shear layer extrusion for the oblique $\left(45^{\circ}\right)$ case is around $1 d$ as observed by the wake structure near the base of the plate in figure 10b. The shape of this dominant structure before roll-up is elliptical rather than circular simply because of the flow angle of incidence.

\section{Results and Discussion for Coherent Inlet Cases}

One now selects and analyzes from the range of simulations conducted, a reference incident coherent gust case chosen at the central values of the limits $A_{\text {ref }}, T_{\text {ref }}[0.5,2.25]$. The non-dimensional numerical time step $\left(\Delta t^{+}=\Delta t U_{0} / d\right)$ for this case is 0.00225 , where $\Delta t=T_{\text {ref }} / 1024$. All coherent inlet gust simulations were restarted from a fully developed uniform inlet case and were run for at least 60 complete incident gust periods (averaged over the last 40). A comparison of the resulting mean stream-wise velocity profile $\left(\bar{U} / U_{0}\right)$ in the wake centerline for this reference case $\left(A_{r e f}, T_{r e f}\right)$ is shown in figure $11 \mathrm{a}$. It is observed that for the $\left(A_{r e f}, T_{r e f}\right)$ test case, the mean recirculation length $\left(L_{R} / d\right)$ is 2.37 , which is about $26 \%$ longer than the uniform inlet case. The maximum velocity deficit is also increased to $0.56 U_{0}$, approximately $27 \%$ higher than in the uniform inlet case. The mean velocity streamline pattern for the $\left(A_{r e f}, T_{r e f}\right)$ test case in the central $(X Y)$ plane presented in figure $11 \mathrm{~b}$ shows that the flow behavior in the mean sense, is similar to the uniform inlet case, where one observes the formation of the two perfectly symmetrical vortices in the plate wake; again confirming sufficient time averaging.

The mean stream-wise $\left(\bar{U} / U_{0}\right)$ and cross-stream $\left(\bar{V} / U_{0}\right)$ velocity profile comparisons between the $A_{\text {ref }}, T_{\text {ref }}$ and the uniform inlet case at various downstream locations are shown in figure 12 . For the uniform inlet case, the mean stream-wise $\left(\bar{U} / U_{0}\right)$ velocity along the wake centerline shows a complete recovery of the deficit at $X / D=2.0$. However, for the $A_{\text {ref }}, T_{\text {ref }}$ gust case, the free stagnation point is located further downstream $(X / D=2.37)$, as also seen by the streamline pattern of figure 11b. Moving downstream in figure $12 \mathrm{a}$, one further observes that for the $A_{\text {ref }}, T_{\text {ref }}$ gust case, the velocity recovery is delayed as a whole. This is also visible from the lower slope of the velocity profile along the wake centerline in figure 11a. On the other hand, the maximum mean cross-stream $\left(\bar{V} / U_{0}\right)$ velocity for the $A_{r e f}, T_{r e f}$ gust case is significantly lower than that of the uniform inlet case (see figure 12b). In fact, moving downstream, one observes 
(a)

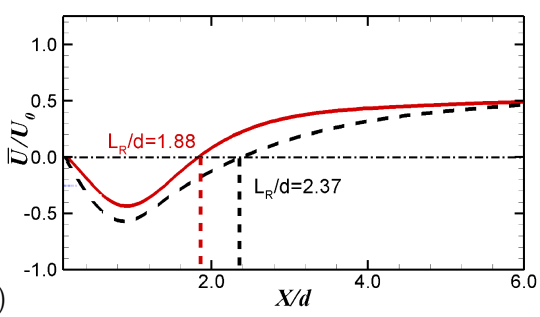

(b)

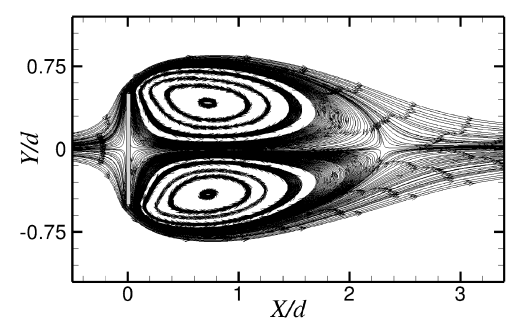

Figure 11: - uniform inlet and - - coherent reference inlet wake velocities. (a) Wake center line mean stream-wise velocity $\left(\bar{U} / U_{0}\right)(\mathrm{b})$ Time averaged velocity streamlines in the wake.

(a)
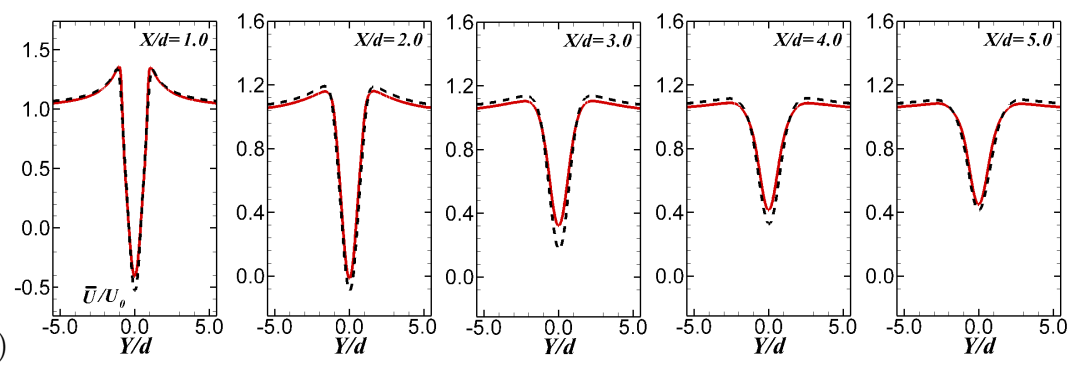

(b)
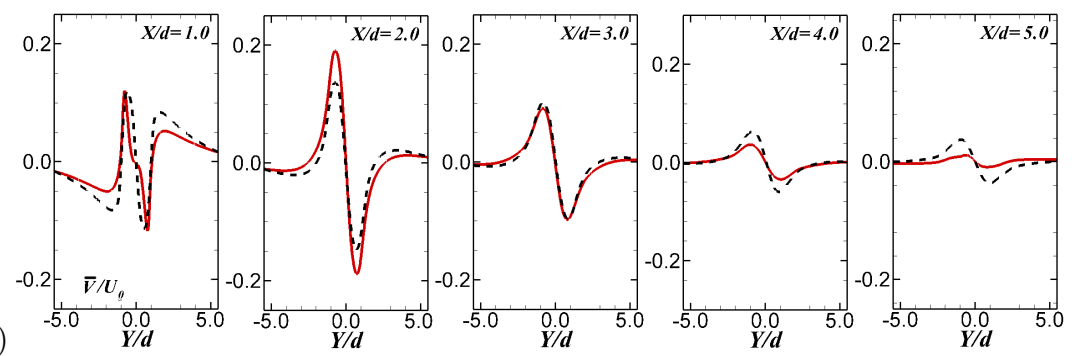

Figure 12: - uniform inlet and -- coherent reference inlet (a) Mean wake profiles for stream-wise velocity $\left(\bar{U} / U_{0}\right)$. (b) Mean wake profiles for cross-stream velocity $\left(\bar{V} / U_{0}\right)$.

that the cross-stream $\left(\bar{V} / U_{0}\right)$ velocity for the two cases exactly overlap at $X / d=3.0$. Furthermore, both upstream and downstream of this location, the $A_{r e f}, T_{r e f}$ gust case shows a delayed increase and a delayed recovery, compared to the uniform inlet case. A comparison of Reynolds normal and shear stress profiles for the two cases along the free stagnation point location $(X / d=2.0)$ are shown in figure 13. Once again, it is observed that the stream-wise $\left(\overline{u^{\prime} u^{\prime}} / U_{0}^{2}\right)$ and span-wise $\left(\overline{w^{\prime} w^{\prime}} / U_{0}^{2}\right)$ fluctuations are slightly higher for the $A_{\text {ref }}, T_{\text {ref }}$ gust case, whereas, the cross-stream $\left(\overline{v^{\prime} v^{\prime}} / U_{0}^{2}\right)$ fluctuations are substantially lower (observe the maximum cross-stream fluctuation level of approximately $0.48 U_{0}$ for the $A_{\text {ref }}, T_{\text {ref }}$ gust case in figure 13$)$. The delay in cross-stream $\left(\bar{V} / U_{0}\right)$ velocity and its substantially lower fluctuation levels $\left(\overline{v^{\prime} v^{\prime}} / U_{0}^{2}\right)$ are owed to the development of additional smaller vortices which are simultaneously shed from the sides of the plates in the $A_{\text {ref }}, T_{\text {ref }}$ gust case; this flow behavior is shown in appendix B.1

For the $A_{\text {ref }}, T_{\text {ref }}$ gust case, instantaneous lift $\left(C_{L}\right)$ and $\operatorname{drag}\left(C_{D}\right)$ responses for approximately 32 gust cycles are shown in figures $14 \mathrm{a} \& \mathrm{~b}$, respectively. In figure $14 \mathrm{c}$, a zoomed in instantaneous drag $\left(C_{D}\right)$ response is shown for a single inlet gust cycle starting at time $90 \mathrm{~s}$. The positive peaks in this figure are labeled as $P_{i, N}$ and the negative ones 

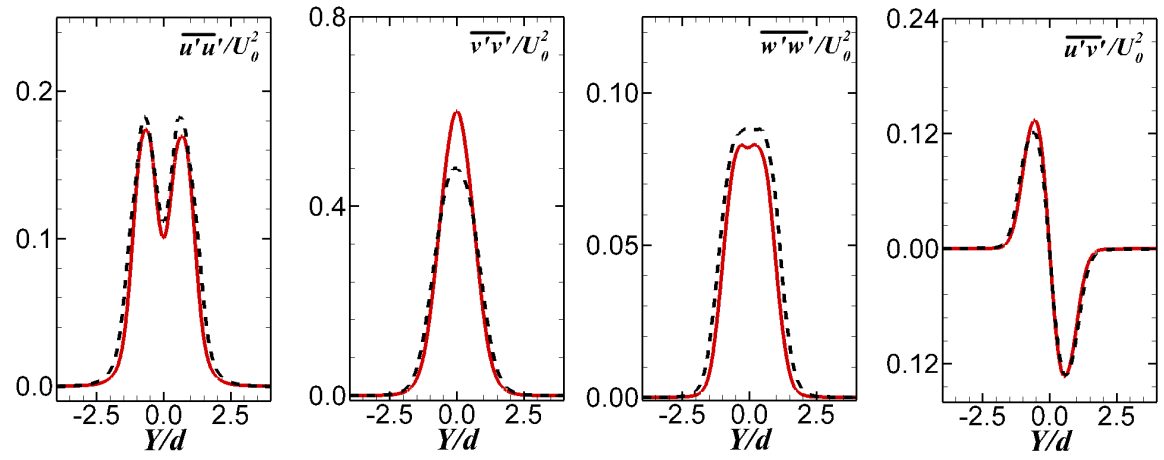

Figure 13: Comparisons between - uniform inlet and -- coherent reference inlet Reynold stresses at $X / d=2.0$ downstream of the flat plate.

(a)

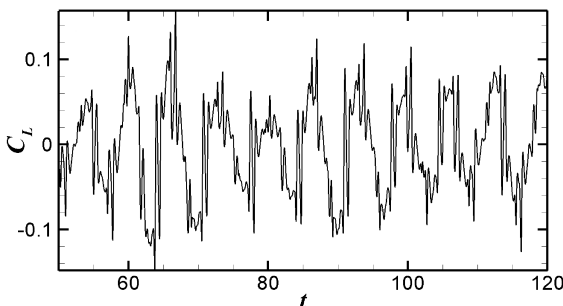

(b)

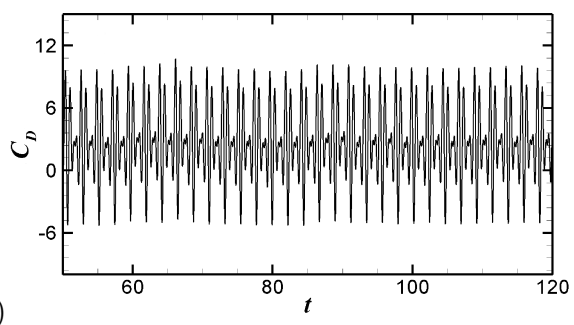

(c)

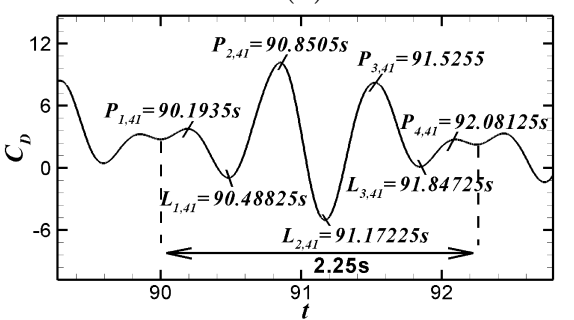

Figure 14: Aerodynamic force responses of the flat plate under coherent reference inlet conditions $[A, T]=[0.5,2.25]$ (a) Instantaneous lift $\left(C_{L}\right)$ (b) Instantaneous drag coefficient $\left(C_{D}\right)(\mathrm{c})$ Zoomed in instantaneous drag coefficient $\left(C_{D}\right)$ for a single gust cycle $(N=41)$.

are labeled as $L_{i, N}$, where $i$ is the peak number and $N$ is the gust cycle number; in the current plot $N=41$. At a glance, the instantaneous drag $\left(C_{D}\right)$ response looks exactly like the inlet gust profile (see the profile shape of a single gust in figure 15a), with an additional maxima peak $\left(P_{4, N}\right)$. However, the global drag cycle shown in figure $14 \mathrm{c}$ corresponds exactly to the time period of the inlet gust signal $(2.25 \mathrm{~s})$. In figure $14 \mathrm{c}$, one observes the two highest peaks of the drag $\left(C_{D}\right)$ coefficient located at $P_{2,41}=90.8505 \mathrm{~s}$ and $P_{3,41}=91.5255 \mathrm{~s}$. For any incident coherent gust case, one could in principle define the location of these peaks in a non-dimensional $\left(P n_{i, N}\right.$ or $\left.L n_{i, N}\right)$ form as

$$
\begin{aligned}
& P n_{i, N}=\left(\frac{P_{i, N}-(N-1) \times T}{T}\right) \times 100 \\
& L n_{i, N}=\left(\frac{L_{i, N}-(N-1) \times T}{T}\right) \times 100
\end{aligned}
$$


(a)

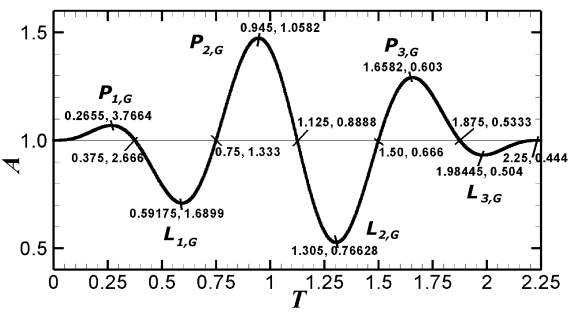

(c)

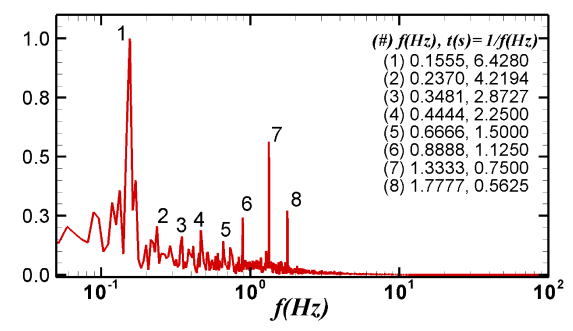

(b)

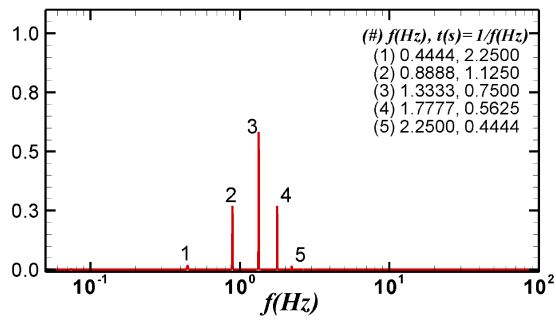

(d)

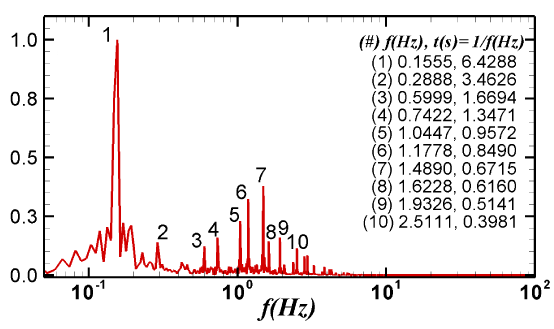

Figure 15: (a) Coherent reference inlet gust with $[A, T]=[0.5,2.25]$. (b) PSD of the inlet pulse for 60 gust cycles. (c) PSD of the stream-wise velocity component in the wake of the flat plate for a probe located at $X / d=1.0 \& Y / d=0.5$. (d) PSD of lift signal of the flat plate.

where $P n_{i, N}$ or $L n_{i, N}$ is the non-dimensional location, $P_{i, N}$ or $L_{i, N}$ is the peak location, $i$ is the peak number, $N$ is the number of cycles or periods of gust and $T$ is the time period of the gust. For the $A_{\text {ref }}, T_{\text {ref }}$ gust case, the non-dimensional positive peaks two $\left(P n_{2,41}\right)$ and three $\left(P n_{3,41}\right)$ then become $37.8 \%$ and $67.8 \%$, respectively. Similarly, for the inlet gust signal (shown in figure 15a), one observes the global $\left(P_{2, G}\right)$ and local $\left(P_{3, G}\right)$ maxima locations at $0.945 \mathrm{~s}$ and $1.658 \mathrm{~s}$, respectively, where the subscript $G$ identifies that the associated signal is that of the inlet gust. Now by using equations $5.1 \mathrm{a} \& \mathrm{~b}$, one obtains their non-dimensional counterparts as $P n_{2, G}=42 \%$ and $P n_{3, G}=73 \%$. Now the difference between the corresponding non-dimensional peaks between the inlet gust signal and the coherent reference case for any cycle can simply be calculated as

$$
\begin{gathered}
\Delta P n_{i, N}=P n_{i, G}-P n_{i, N} \\
\Delta L n_{i, N}=L n_{i, G}-L n_{i, N}
\end{gathered}
$$

For example, for the gust cycle 41 , for the $A_{\text {ref }}, T_{\text {ref }}$ gust case, the non-dimensional global $\left(\triangle P n_{2,41}\right)$ peak difference comes out to be $4.2 \%$ and the non-dimensional local $\left(\Delta L n_{3,41}\right)$ peak difference comes out to be $5.2 \%$. Similar comparisons were made for a number of different cycles for the $A_{\text {ref }}, T_{\text {ref }}$ gust case and it was found that the nondimensional difference in any maxima or minima peak never exceeds $6 \%$ as can be seen from table 4 . Similar observations were made regarding the maxima and minima peaks for all other coherent inlet cases and hence it was concluded that the lower frequencies (or larger structures) for any coherent inlet simulation exhibit a very similar response. This idea leads to the formulation of drag reconstruction, where one could in principle take the inlet gust formulation and the velocity record of a coherent inlet simulation and then predict the instantaneous drag response of another case with a similar coherent inlet. This is shown in detail later in section 6 . There still remains one maxima peak $\left(P_{4,41}\right)$, which can be seen in figure $14 \mathrm{c}$, which is additional to the oscillations of the inlet. The existence of this additional peak in the drag response was seen to be consistent throughout all the 


$\begin{array}{cccccccc}\mathbf{N} & \Delta P n_{1, N} & \Delta P n_{2, N} & \Delta P n_{3, N} & \Delta L n_{1, N} & \Delta L n_{2, N} & \Delta L n_{3, N} & P_{4, N} \\ 31 & 4.2 & 4.5 & 5.0 & 2.3 & 4.7 & 5.3 & 92.9 \\ 41 & 3.2 & 4.2 & 5.2 & 1.6 & 5.9 & 6.0 & 92.5 \\ 60 & 4.0 & 5.5 & 4.6 & 3.4 & 5.2 & 5.7 & 92.4\end{array}$

Table 4: The non-dimensional difference in the maxima and minima peaks between the coherent reference $A_{r e f}, T_{r e f}$ gust case and inlet gust signal at various gust cycles $(N=$ $31,41,60)$.

tested coherent inlet cases. For the reference $A_{\text {ref }}, T_{\text {ref }}$ incident coherent gust case, the non-dimensional location of this maxima peak is between $92-93 \%$ (see the last column of table 4 .

The power spectrum density ( $P S D)$ of the inlet gust profile for the $A_{\text {ref }}, T_{\text {ref }}$ test case is shown in figure 15b. The $P S D$ is taken from Fourier Transform of the velocity record of a probe far upstream of the flat plate for complete 60 gust cycles. As a validation, the spectrum of the inlet profile for 60 cycles was also plotted separately and no difference was found in the two curves. The $P S D$ in all the figures $15 \mathrm{~b}, \mathrm{c} \& \mathrm{~d}$, is normalized by the magnitude of the most energetic frequency downstream of the flat plate (which is actually the non-dimensional Strouhal number of the plate). On each of the figures 15b, $\mathrm{c} \& \mathrm{~d}$, the most energetic peaks are numbered and displayed on the top right in the form of location number (\#), frequency $(f(H z))$ \& time $(t(s)=1 / f(H z))$. Closely observing the inlet gust signal in figure $15 \mathrm{a}$ and its spectrum profile in figure $15 \mathrm{~b}$, one notices that the inlet gust is actually a combination of a number of sine waves with changing amplitude. The frequencies identified as 1 and 5 are associated to the combined effect of the entire gust signal as $1 / f_{1}$ leads to the time period of the gust $(t=2.25 \mathrm{~s})$ and can be ignored or filtered out of the signal. The frequencies identified as 2 and 4 are due to half and quarter gust periods. Whereas, the most energetic frequency observed in figure 15b, is at location 3 (at $f=1.3333 \mathrm{~Hz}$ or $t=1.125 \mathrm{~s}$ ), which is actually one third the gust wave period. In figure $15 \mathrm{c}$, the spectrum of the stream-wise velocity signal of a probe downstream of the flat plate (at $X / d=1.0 \& Y / d=0.5$ ) is shown. It is observed that the normal flat plate under the coherent reference incident gust, exhibits a primary or most energetic frequency (also the Strouhal number) at location $1(f=0.15555$ ), which is slightly lower than the Strouhal number of a flat plate with uniform inlet (0.1666). The three high peaks of the superimposed gust signal are still seen with little or no loss of the signal strength at locations $6,7 \& 8$. The spectrum of the instantaneous lift signal of the flat plate for the same $A_{\text {ref }}, T_{\text {ref }}$ gust case is shown in figure $15 \mathrm{~d}: 1^{\text {st }}, 2^{\text {nd }}$ and $3^{\text {rd }}$ peaks correspond to the primary, secondary and tertiary frequencies of the flat plate with uniform inlet (same as in figure 6c), whereas the additional peaks are either higher harmonics and/or constructive and destructive interference due to phase and amplitude differences between multiple wave fronts which combine to define a single gust signal. Time evolution of the complex vortex shedding in the wake of the plate for the same coherent $A_{\text {ref }}, T_{\text {ref }}$ reference incident gust case is presented in appendix B.1 for three complete gust cycles. The shedding cycle time monitored by time evolution of the wake leads to almost the same shedding frequency of $f \approx 0.153$ which is almost the same as the global primary shedding frequency of 0.155 . One could in principle perform a phase averaging based on the period of oscillations of the incident gust. However, as the frequency of the inlet perturbations is much higher than the shedding frequency of the 
bluff body this will essentially washout the underlying flow physics. Thus, the results for all current simulations are presented only averaged in time

\section{Drag Reconstruction}

Till now, we have looked at the response and wake behavior of a flat plate with uniform and unsteady incident coherent gusts. We now focus on quantifying the level of admittance or simply the disturbances which are added to the system with the inlet unsteadiness. This not only explains how the flow physics changes with upstream perturbations (strictly speaking not looking at small scale inlet turbulent perturbations) but also enables one to quantify the level of unsteadiness thereby enabling the prediction of the bluff body behavior as a whole. Thus, the current section deals with the fluctuating force measurements and drag reconstruction for the normal $\left(90^{\circ}\right)$ flat plate under the effect of incident coherent velocity fluctuations. The methodology for selection and implementation of the gust velocity profiles and the flow behavior have already been discussed in section 2. Uniform steady flow over an object moving in-line with the flow is essentially equivalent to unsteady stream-wise fluctuations over a stationary object, provided that the magnitude of the oscillations of the former (object) are equal to the stream-wise fluctuations of the latter (flow); Lighthill (1954). However, relative to the incoming flow, the body experiences an acceleration or a deceleration, leading to an added virtual mass which is basically the inertia added-to or subtracted-from the system due to the acceleration or deceleration. For such unsteady flows, there exists a strong and direct relationship between the flow fluctuations and the stream-wise forces acting on the body. This relationship is generally represented in the form of a frequency-dependent transfer function, called the aerodynamic admittance function or simply admittance (see Drabble et al. (1990)). The admittance function not only depends upon the nature of the flow instabilities but also on the geometric shape of the body. Furthermore, it is generally accepted that in a fully turbulent flow the admittance decreases if the frequency of the velocity fluctuations increase. On the other hand, for flows with coherent fluctuations, admittance increases directly as the frequency of the velocity fluctuations increase. For such coherent flows, admittance is defined by Vickery (1965) as

$$
X^{2}(n)=1+\left(\frac{C_{m}}{C_{D}}\right)^{2}\left(\frac{2 \pi n d}{\bar{U}}\right)^{2}
$$

where $C_{D}$ is the drag coefficient, $C_{m}$ the virtual or added mass coefficient, $n$ the frequency of the fluctuations, $d$ the characteristic length scale and $\bar{U}$ the mean flow velocity. However, a more general representation in a non-dimensional form given by Drabble et al. (1990) reads as

$$
X^{2}(n)=\frac{\bar{U}^{2}}{4 \bar{F}^{2}}\left(\frac{S_{F}(n)}{S_{u}(n)}\right)
$$

where $\bar{F}$ is the mean stream-wise force acting on the body, $S_{F}(n)$ the power spectral density of the fluctuating force and $S_{u}(n)$ the power spectral density of the velocity fluctuations upstream of the flat plate. For fully coherent fluctuations, the admittance and aerodynamic response of the body is generally estimated by the Lattice theory developed by Vickery (1965), later deeply analyzed by Bearman (1971). The predictions of the lattice theory are valid only for configurations where the turbulent aspect ratio (defined as the ratio of the longitudinal integral scale of turbulence to the characteristic length scale) is sufficiently high (greater than 1.5). Further limitations lie in the fact that to apply the lattice theory both the shape and the length scale of turbulence need to be known a-priori. 
(a)

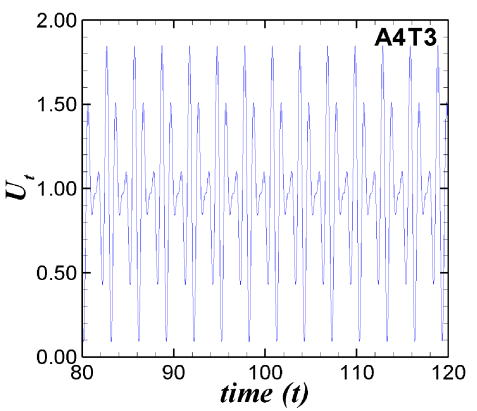

(b)

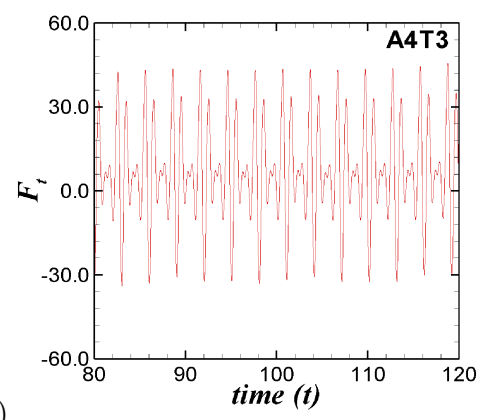

Figure 16: Incident coherent gust case A4T3 (a) Instantaneous stream-wise fluctuating velocity $\left(U_{t}\right)$ profile at inlet (upstream of the plate) (b) Computed fluctuating force response in the stream-wise direction $\left(F_{t}\right)$.

(a)

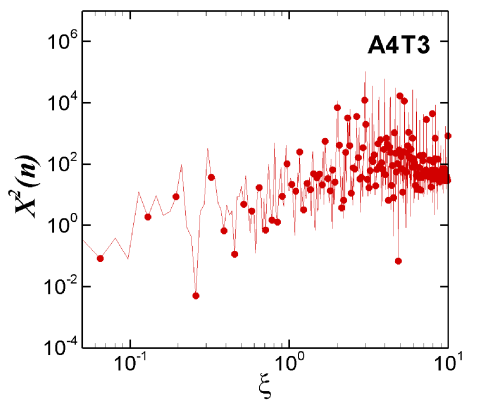

(b)

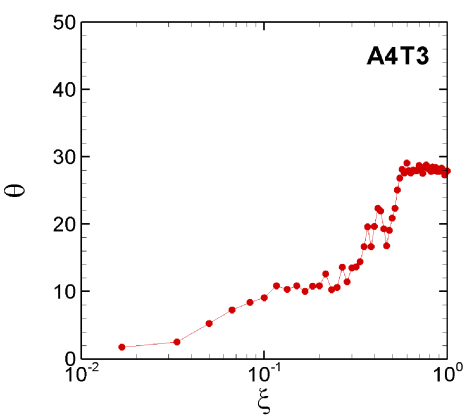

Figure 17: Incident coherent gust case A4T3 (a) Admittance coefficient $\left(X^{2}(n)=\right.$ $\left.\left(\bar{U}^{2} / 4 \bar{F}^{2}\right)\left(S_{F}(n) / S_{u}(n)\right)\right)$ (b) Phase angle between the fluctuating force and the fluctuating velocity against the non-dimensional frequency $(\xi=n d / \bar{U})$.

$\begin{array}{ccccc}\text { Cases } & A_{1}(0.0694318) & A_{2}(0.3300094) & A_{3}(0.6699905) & A_{4}(0.9305681) \\ T_{1}(0.3124432) & 2.3133 & 2.3523 & 2.4907 & 2.5640 \\ T_{2}(1.4850426) & 2.3672 & 2.4382 & 2.5133 & 2.6091 \\ T_{3}(3.0149573) & 2.3681 & 2.5010 & 2.2530 & 2.6247 \\ T_{4}(4.1875567) & 2.3709 & 2.5410 & 2.5565 & 2.6538\end{array}$

Table 5: Mean drag $\left(\bar{C}_{D}\right)$ calculated for the normal $\left(90^{0}\right)$ flat plate with coherent velocity inlets.

To solve this dilemma, the general approach of assuming the turbulence to be isotropic, leads to predictions which are quite crude (see Drabble et al. (1990)). Thus, for the current study, one analyzes the coherent inlet flow fluctuations with the aid of a methodology presented by Drabble et al. (1990). The aerodynamic admittance is computed along with the transient drag reconstruction on the basis of the frequency dependent drag $C_{D}(\xi)$ and virtual mass $C_{m}(\xi)$ coefficients, where $\xi$ is the non-dimensional frequency defined as $n d / \bar{U}$.

The 16 cases outlined in table 5 were restarted from a fully developed field using the results of the uniform inlet case. The resulting computed mean drag $\left(\bar{C}_{D}\right)$ coefficients for 
(a)

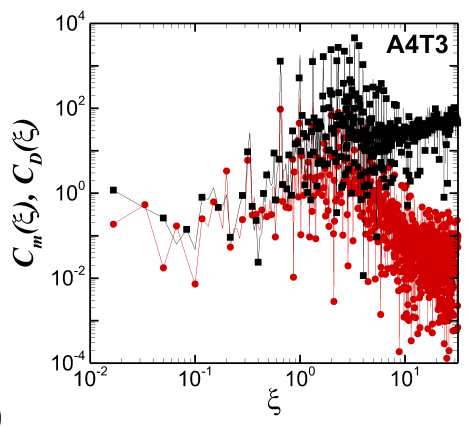

(b)

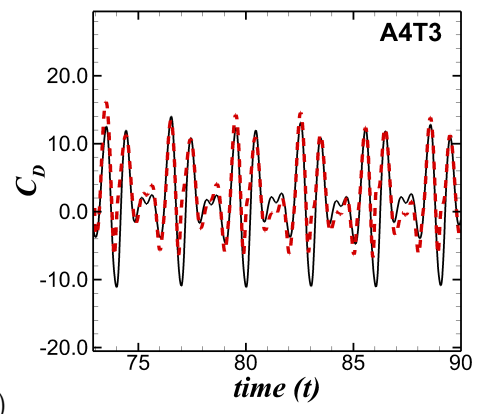

Figure 18: Incident coherent gust case A4T3 (a) Frequency dependent virtual mass $C_{m}(\xi)$-lower profile) and drag $\left(\boldsymbol{\square} C_{D}(\xi)\right.$-upper profile) coefficients (b) Comparisons between the $(-)$ computed and $(--)$ predicted drag $\left(C_{D}\right)$ profiles using only the highest fundamental frequency of the actual velocity signal.

each case are listed in table 5 . It is interesting to note from table 5 that the mean drag is directly dependent, not only on the amplification of the coherent velocity, but also on the time period of inlet gust cycle. Even-though, the mean drag $\left(\bar{C}_{D}\right)$ shows a general increasing trend for higher amplitude of oscillations, the cases with smaller time periods show high degree of r.m.s. values (not tabulated in this paper). In order to measure the velocity fluctuations, a number of probes were selected; upstream, on the side and in the wake of the plate. The instantaneous velocity signals for a probe upstream of the body for incident coherent gust case A4T3 is shown in figure 16a. The resulting force response in the stream-wise direction to the inlet velocity signal is reported in figure 16b. From the trend of the velocity fluctuations and the force response it can be safely assumed that the fluctuations are fully coherent as per the adopted inlet formulation.

For all practical purposes for the calculation of admittance coefficient $\left(X^{2}(n)=\right.$ $\left.\left(\bar{U}^{2} / 4 \bar{F}^{2}\right)\left(S_{F}(n) / S_{u}(n)\right)\right)$ experimentally, the spectrum of the velocity fluctuations $\left(S_{u}(n)\right)$ is taken from a case in the absence of the plate model with no turbulent or coherent fluctuations in the flow. For the current study, the spectrum of velocity fluctuations from a probe upstream of the plate from the uniform inlet simulation is used instead. The spectrum for the fluctuating force $\left(S_{F}(n)\right)$, on the other hand is calculated from the actual computed stream-wise force response for the coherent inlet cases. The computed admittance coefficients for A4T3 case is shown in figure 17a. It is observed, that for the coherent fluctuations, the admittance coefficient shows an increasing trend with an increase in the non-dimensional frequency, as reported by Drabble et al. (1990).

In the current cases, the coherence spectrum (as a function of non-dimensional frequency between, the fluctuating velocity signal at a probe upstream of the flow and the fluctuating stream-wise force) is utilized in identifying the identical fundamental frequencies. The phase angle $(\theta)$ is then computed through the phase spectrum between the two (velocity and force response) signals for A4T3 case and is shown in figure 17b. Since the phase angle is now a sort of measure between the inertial and drag forces, it shows an increasing trend with an increase in the non-dimensional frequency. It then follows from figure 17, that for small phase $(\theta)$ angles, the drag component is dominant whereas for large $(\theta)$ the inertial component is dominant. This is logically true since this means that when the flow structures are large, their effect on both the velocity signal and the drag response is similar leading to a closer coherence and a smaller phase difference between them. On the other hand, when the inertial components are large, the scales are smaller 
depicting a larger phase angle difference between the force response in the shape of drag profile and the velocity signal. Similar behavior is observed and reported by Drabble et al. (1990).

For fully coherent fluctuations as reported by Drabble et al. (1990), the total streamwise force acting in the plate can be represented as

$$
F(t)=\frac{1}{2} \rho d^{2} C_{D} u^{2}(t)+\rho d^{3} C_{m} \frac{d u(t)}{d t}
$$

where the velocity signal $(u(t))$ can be decomposed into its mean $(\bar{U})$ and fluctuating $\left(u^{\prime}\right)$ parts. The same concept when exploited for the total force leads to the decomposition of the total drag force $(F(t))$ into its mean $(\bar{F})$ and fluctuating $(\widetilde{F})$ components as

$$
\begin{aligned}
& \bar{F}=\frac{1}{2} \rho d^{2} C_{D}\left(\bar{U}^{2}+\overline{\left(u^{\prime}\right)^{2}}\right) \\
& \widetilde{F}=\rho d^{2} C_{D} \bar{U} u^{\prime}+\rho d^{3} C_{m} \frac{d u^{\prime}}{d t}
\end{aligned}
$$

where equation $6.4 \mathrm{~b}$ shows the contribution from both stream-wise and inertial components of the force in the form of $C_{D}$ and $C_{m}$ respectively. Unfortunately, as pointed out by Davenport (1961) and Davenport (1967), the drag component in equation 6.4a needs to be the smooth drag $\left(C_{D s}\right)$ in the absence of any fluctuations i.e. for uniform inlet flow. However, the drag $\left(C_{D}\right)$ and virtual mass $\left(C_{m}\right)$ components in equation $6.4 \mathrm{~b}$ are frequency dependent as they are associated with the fluctuating forces i.e. $\left(C_{D}(\xi)\right)$ and $\left(C_{m}(\xi)\right)$ respectively. Thus, the true representation of the mean and fluctuating force components should be

$$
\begin{gathered}
\bar{F}=\frac{1}{2} \rho d^{2} C_{D s}\left(\bar{U}^{2}+\overline{\left(u^{\prime}\right)^{2}}\right) \\
\widetilde{F}=\rho d^{2} C_{D}(\xi) \bar{U} u^{\prime}+\rho d^{3} C_{m}(\xi) \frac{d u^{\prime}}{d t}
\end{gathered}
$$

In light of equations $6.5 \mathrm{a} \& \mathrm{~b}$, the admittance as a function of frequency dependent drag $\left(C_{D}(\xi)\right)$ and virtual mass $\left(C_{m}(\xi)\right)$ coefficients then becomes

$$
X^{2}(n)=\frac{C_{D}^{2}(\xi)}{C_{D s}^{2}}\left[1+\left(\frac{2 \pi n d}{\bar{U}}\right)^{2} \frac{C_{m}^{2}(\xi)}{C_{D}^{2}(\xi)}\right]
$$

whereas the phase angle between the fluctuating velocity and the fluctuating force becomes

$$
\theta(n)=\tan ^{-1}\left[\frac{C_{m}(\xi)}{C_{D}(\xi)}\left(\frac{2 \pi n d}{\bar{U}}\right)\right]
$$

Now, since the admittance $\left(X^{2}(n)\right)$ from equation 6.2 , smooth drag coefficient $\left(C_{D s}\right)$ from a separate uniform inlet simulation and phase angle $(\theta)$ from coherence and phase spectrum are already known, one can compute the frequency dependent $\operatorname{drag}\left(C_{D}(\xi)\right)$ and virtual mass $\left(C_{m}(\xi)\right)$ coefficients from equations $6.6 \mathrm{a} \& \mathrm{~b}$. These frequency dependent $C_{D}(\xi)$ and $C_{m}(\xi)$ are shown against the non-dimensional frequency $(\xi=n d / \bar{U})$ for A4T3 case in figure 18a. As expected, the drag $\left(C_{D}(\xi)\right)$ and added mass or inertial $\left(C_{m}(\xi)\right)$ components show an opposite trend with increasing non-dimensional frequency.

The stage is now set to predict or reconstruct the instantaneous drag response for the coherent inlet cases. This is done by choosing a fundamental (most energetic) frequency, in the current case, a frequency close to the inlet oscillation time period, recording the values of $\left(C_{D}(\xi)\right)$ and $\left(C_{m}(\xi)\right)$ at this frequency, and by using the appropriate velocity record of a separate coherent inlet simulation to solve equation $6.5 \mathrm{~b}$. The predicted 
fluctuating drag component for the case under discussion (A4T3) is shown in figure 18b. The reconstructed mean drag for the A4T3 $\left(C_{D p}=2.458\right)$ case was found to be in good agreement with the computed mean drag coefficient $C_{D}=2.6247$. Interestingly, not only the mean drag $\left(\bar{C}_{D}\right)$ values but also the instantaneous profiles are predicted quite accurately by only using a single fundamental frequency. One could in theory use multiple frequencies or even the entire velocity record of another simulation to improve the drag reconstruction. The most obvious advantage of drag reconstruction is that it can in principle be used as an uncertainty quantification tool to predict the fluctuating force response, of a similar object subjected to coherent fluctuations or even to the same configuration at a different time instant.

\section{Conclusions}

Flow over a normal $\left(90^{\circ}\right)$ and an oblique $\left(45^{0}\right)$ flat plate was numerically tested with a uniform inlet. The normal $\left(90^{\circ}\right)$ case was also simulated with incident coherent velocity fluctuations. For the uniform inlet cases, the results were found to be in excellent agreement with the DNS and experimental data. For the normal $\left(90^{\circ}\right)$ uniform inlet case, alternate coherent vortex shedding was observed, whereas for the oblique $\left(45^{0}\right)$ uniform inlet case, the shedding from the two ends of the plate interacted strongly resulting in a quasi-periodic force response where the flow exhibited multiple frequencies in the wake. It is thus concluded that the complex wake behind oblique or inclined bluff bodies substantially differs from that of a normal bluff body where the thickness of the bluff body plays a key role.

The second half of the paper presented results for the cases with fully coherent incident gusts where both the admittance and mean drag were computed. It is concluded that when the period of oscillation is short with high levels of amplitude of oscillations, the r.m.s fluctuations of the drag coefficient are higher. However, the mean drag and lift coefficients still show an increasing trend with an increase in the amplitude and time period of the oscillations. An attempt was made for the drag reconstruction following the method of Drabble et al. (1990) for some of the cases. The reconstructed transient drag and its mean were found to be in good agreement with the computed drag coefficient. It is thus concluded that one could in principle use the current methodology to predict with reasonable accuracy, the response of a similar bluff body with unsteady incident coherent gusts. From an engineering perspective, this makes a novel contribution to the field of fluid mechanics, where one not only needs to access the wake behavior under unsteady conditions, but also needs a formal tool or basis for its response prediction.

\section{Acknowledgments}

The authors would like to acknowledge the funding from French National Research Agency $(A N R, S T U R M-4)$ and the continued funding by UK Research Council (EP$S R C$, Computations for Advanced Reactor Engineering (CARE). We are also thankful to Électricité de France (EDF) for the computational resources and to Dr. V. D. Narasimhamurthy for providing the DNS data for comparisons.

\section{REFERENCES}

Afgan, I., Moulinec, C., Prosser, R. \& Laurence, D. 2007. Large Eddy Simulation of turbulent flow for wall mounted cantilever cylinders of aspect ratio 6 and 10. Int. J. of Heat and Fluid Flow. 28, pp. 561-574. 
Afgan, I., Kahil, Y., Benhamadouche, S. \& Sagaut, P.2011. Lareg Eddy Simulation of the flow around single and two side-by-side cylinders at subcritical Reynolds numbers. Physics of Fluids. 23, 075101.

Archambeau, F., Mchitoua, N., \& Sakiz, M. 2004. Code_Saturne : A finite volume code for the computation of turbulent incompressible flows-industrial applications. Int. J. of Finite Vol. 1. ISSN 1634(0655).

Bearman, P. W. 1971. Investigation of forces on flat plate normal to a turbulent flow. J. Fluid. Mech. Vol.46, pp. 177-198.

Benhamadouche, S., Laurence, D., Jarrin, N., Afgan, I. \& Moulinec, C. 2005. Large Eddy Simulation of flow across in-line tube bundles. In Nuclear Reactor Thermal Hydraulics, NURETH-11. Avignon, France. Paper 405.

Bierbooms, W. \& Cheng, P-W. 2002. Stochastic gust model for design calculation of wind turbine. J. Wind Eng. Ind. Aerodyn. 90, pp. 1237-1251.

Bierbooms, W. 2004. A gust model for wind turbine design. JSME Int. J. Series B. 47(2), pp. $378-386$

Breutr, M. \& Jovicic, N. 2001. Separated Flow Around a Flat Plate at High Incidence:An LES Investigation. J. of Turb. vol 2, No. 18.

Chen, J. M. \& FAng, Y. -C. 1996. Strouhal numbers of inclined flat plates. J. Wind Engg. \&6 Indust. Aero. 61, pp. 99-112.

Dennis, S. C. R., Wang-Qiang, Coutanceau, M. \& Launay, J. L. 1993. Viscous flow normal to a flat plate at moderate Reynolds numbers. J. Fluid Mech. 248, pp. 605-635.

Davenport, A. G. 1961. The application of statistical concepts to the wind loading of structures. Proc. Inst. Civ. Engrs. 19, pp. 449-472.

Davenport, A. G. 1967. Gust loading factors. J. Struct. Div. 93(3), pp. 11-34.

Drabble, M. J., Grant, I., Armstrong, B. J. \& Barnes, F. H. 1990. The aerodynamic admittance of a square plate in a flow with a fully coherent fluctuation. Physics of Fluids A. Vol 2(6), pp. 1005-1013.

Fage, A. \& Johansen, F. C. 1927. On the flow of air behind an inclined flat plate of infinite span. In Proceedings of the Royal Society of London. vol 116(A), No. 773. pp. 170-197.

Fox, T. A. \& West, G. S. 1990. On the use of end plates with circular cylinders. Exps. Fluids. 9, pp. 231-239.

Goyette, S., Brasseur, O. \& Beniston, M. 2003. Application of a new wind gust parametrization: Multi-scale case studies performed with the Canadian regional climate model. J. Geophys. Res. 108(D13):4374.

Harper, B. A., Kepert, J. D. \& Ginger, J. D. 2008. Guidelines for converting between various wind averaging periods in tropical cyclone conditions. World Meteorological Organization Report. Appendix II.

Hunt, J. C. R., Wray, A. A. \& Moin, P. 1988. Eddies, stream and convergence zones in turbulent flows. Report CTR-S88. Center for Turbulent Research.

Hussain, A. K. M. F. 1986. Coherent structures and turbulence. J. Fluid Mech. 173, pp. 303356.

Julien, S., Lasheras, J. \& Chomaz, J,-M. 2003. Three-dimensional instability and vorticity patters in the wake of a flat plate. J. Fluid Mech. 479, pp. 155-189.

Julien, S., Ortiz, S. \& Chomaz, J.-M. 2004. Secondary instability mechanisms in the wake of a flat plate. European Journal of Mechanics B/Fluids. 23, pp. 157-165.

Kim, D. H., YAng, K. S. \& Senda, M. 2004. Large eddy simulation of turbulent flow past a square cylinder confined in a channel. Comp. \& Fluids. 33, pp. 81-966.

KiYA, M. \& MATSUmurA, M. 1988. Incoherent turbulence structure in the near wake of a normal plate. J. Fluid Mech. 190, pp. 157-165.

Koumoutsakos, P. \& Shiels, D. 1996. Simulations of the viscous flow normal to an impulsively started and uniformly accelerated flat plate. J. Fluid Mech. 328, pp. 177-227.

Lamont, P. J. \& Hunt, B. L. 1980. The impingement of underexpanded, axisymmetric jets on perpendicular and inclined flat plates. J. Fluid Mech. 100, pp. 471-511.

Leder, A. 1991. Dynamics of fluid mixing in separated flows. Physics of Fluids. 3(7), pp. 17411748.

Lighthill, M. J. 1954. The response of laminar skin friction and heat transfer to fluctuations in the stream velocity. proc. $R$. Soc. London. Series A, 224, pp. 1-23. 
MazharoĞLu, Ç. \& Hacişevki, H. 1999. Coherent and incoherent flow structures behind a normal flat plate. Exp. Thermal and Fluid Science. 19, pp. 160-167.

Moulinec, C., Benhamadouche, S., Laurence, D. \& Peric, M. 2005. LeS in a U-bend pipe meshed by polyhedral cells. In ERCOFTAC ETMM-6 Conference. Elsevier, Sardinia.

Moser, R. \& Balachandar, S. 1998. Self-similarity of time-evolving plane wakes. J. Fluid Mech. 367, pp. 255-289.

NAJJAR, F. M. \& BALACHANDAR, S.1998 . Low frequency unsteadiness in the wake of a normal flat plate. J. Fluid Mech. 370, pp. 101-147.

NAJjAR, F. M. \& VANKA, S. P. 1995a. Simulations of the unsteady separated flow past a normal flat plate. Int. J. of Numerical Methods in Fluids. 21, pp. 525-547.

NAJjar, F. M. \& VANKA, S. P. 1995b. Effects of intrinsic three-dimensionality on the drag characteristics of a normal flat plate. Physics of Fluids. 7(10), pp. 2516-2518.

Nakagawa, S., NitTa, K. \& Senda, M. 1999. An experimental study on unsteady turbulent near wake of a rectangular cylinder in channel flow Exp Fluids. 27(3), pp. 284-294.

Narasimhamurthy, V. D. \& Andersson, H. I. 2009. Numerical simulation of the turbulent wake behind a normal flat plate. Int. J. of Heat and Fluid Flow. 30, pp. 1037-1043.

NorberG, C. 1994. An experimental investigation of the flow around a circular cylinder: influence of aspect ratio. J. Fluid Mech. 258, pp. 287-316.

Norberg, C. 2003. Fluctuating lift on a circular cylinder: review and new measurements $J$. Fluids and Struc. 17, pp. 57-96.

Pagnini, L. C. \& Solari, G. 2002. Gust buffeting and turbulence uncertainties. J. Wind Eng. Indust. Aerodyn. 90, pp. 441-459.

Perry, A. E. \& Steiner, T. R. 1987. Large-scale vortex structures in turbulent wakes behind bluff bodies. Part 1: vortex formation processes. J. Fluid Mech. 174, pp. 233-270.

Press, W. H, Teukolsky, S. A., Vetterling, W. T. \& Flannery, B. P. 1996. Numerical Recipes in Fortran 77: Vol 1: The Art of Scientific Computing. Cambridge University Press. $\mathbf{2}^{\text {nd }}$ edition, ISBN:0-521-43064-X.

Quinn, A. D., Baker, C. J. \& Wright, N. G. 2001. Wind and vehicle induced forces on flat plates-Part 1: wind induced force. J. Wind Eng. Indust. Aerodyn. 89, pp. 817-829.

Rhie, C. \& Chow, W. 1982. A numerical study of the flow past an isolated airfoil with trailing edge separation. J. AIAA. 21, pp. 15251532.

Roshko, A. 1993. Prespectives on bluff body aerodynamics. J. Wing Engg. Indust. Aerodyn. 49, pp. $79-100$.

SAнA, A. K. 2007. Far-wake characteristics of two-dimensional flow past a normal flat plate. Physics of Fluids. 19, 128110.

Solari, G \& Piccardo, G. 2000. Probabilistic 3-D turbulence modeling for gust buffeting of structures. Prob. Eng. Mech. 16, pp. 73-86.

Steiner, T. R. \& Perry, A. E. 1987. Large-scale vortex structures in turbulent wakes behind buff bodies. Part 2: far-wake structures. J. Fluid Mech. 174, pp. 271-298.

Tamaddon-Jahromi, H. R., Townsend, P. \& Webster, M. F. 1994. Unsteady viscous flow past a flat plate orthogonal to the flow. Computers and Fluids. 23(2), pp. 433-446.

Thompson, M. C., Hourigan, K., Ryan, K. \& Sheard, G. J. 2006. Wake transition of two dimensional cylinders and axisymmetric bluff bodies. J. Fluids and Struct. 22, pp. 793-806.

VAn Doormal, J.P., Raithby, G.D. 1984. Enhancements of the SIMPLE method for predicting incompressible fluid flows. Numer. Heat Transfer. 7, pp. 147-163.

VICKERY, B. J. 1965. On the flow behind a coarse grid and its use as a model of atmospheric turbulence in studies related to wind loads in buildings. National Physical Laboratory. Aero Report No. 1143.

Wu, S. J., Miau, J. J., Hu, C. C. \& Chou, J. H. 2005. On low-frequency modulations and three-dimensionality in vortex shedding behind a normal plate. J. Fluid Mech. 526, pp. 117-146.

Yao, Y. F., Thomas, T. G., Sandham, N. D. \& Williams, J. J. R. 2001. Direct numerical simulation of turbulent flow over a rectangular trailing edge. Theor. Comput. Fluid Dyn. 14, pp. 337-358

Yeung, W. W. H. \& Parkinson, G. V. 1997. On the steady separated flow around and inclined flat plate. J. Fluid Mech. 333, pp. 403-413. 
(a)
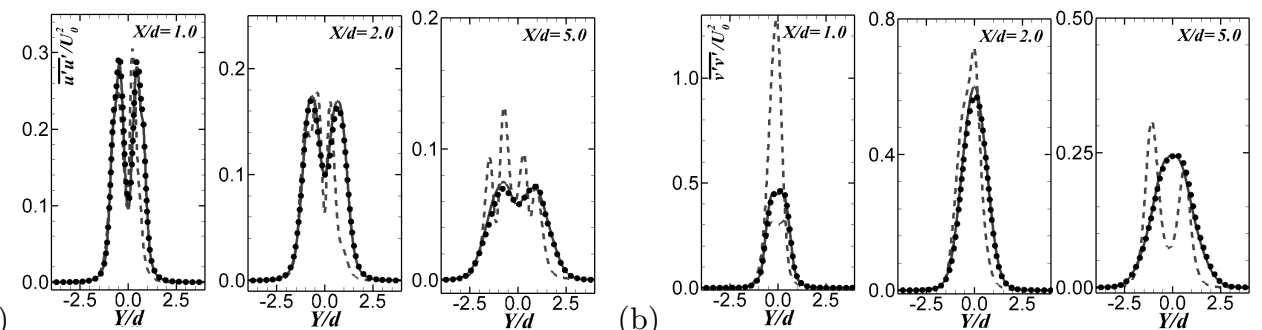

(c)

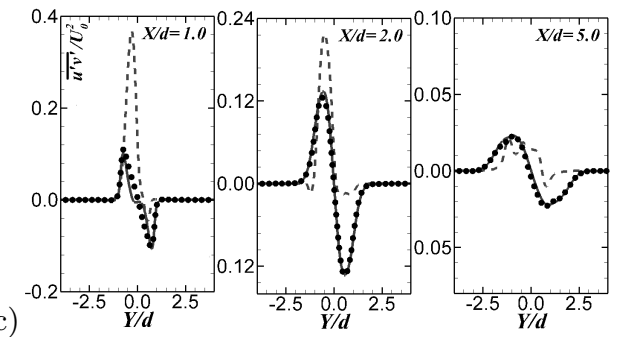

Figure A.1: Mean Reynolds stress profiles ( Narasimhamurthy \& Andersson (2009), - LES normal $\left(90^{\circ}\right)$ and -- LES oblique $\left(45^{\circ}\right)$ configuration) at various downstream locations (a) $\overline{u^{\prime} u^{\prime}} / U_{0}^{2}$ (b) $\overline{v^{\prime} v^{\prime}} / U_{0}^{2}$ (c) $\overline{u^{\prime} v^{\prime}} / U_{0}^{2}$.

(a)

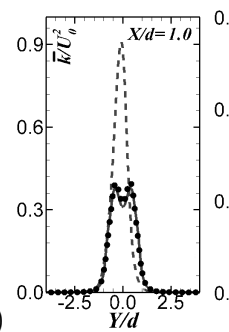

(b)

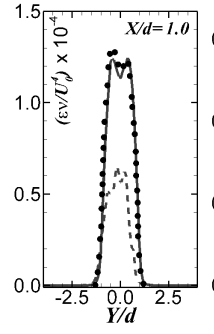

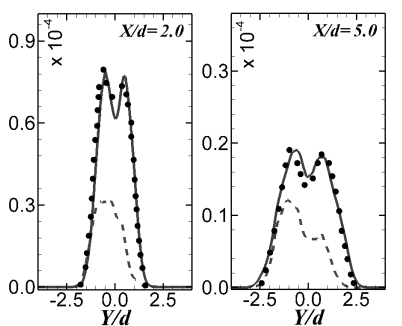

Figure A.2: (a) Turbulent kinetic energy $\left(k / U_{0}^{2}\right)$ (b) Dissipation rate of turbulent kinetic energy $\left(\varepsilon \nu / U_{0}^{4}\right)$ at various downstream locations, Narasimhamurthy \& Andersson (2009), - LES normal $\left(90^{\circ}\right)$ and -- LES oblique $\left(45^{\circ}\right)$ configuration.

Appendix A. Reynolds Stress, Turbulent Kinetic Energy and Dissipation for Uniform Inlet Cases 

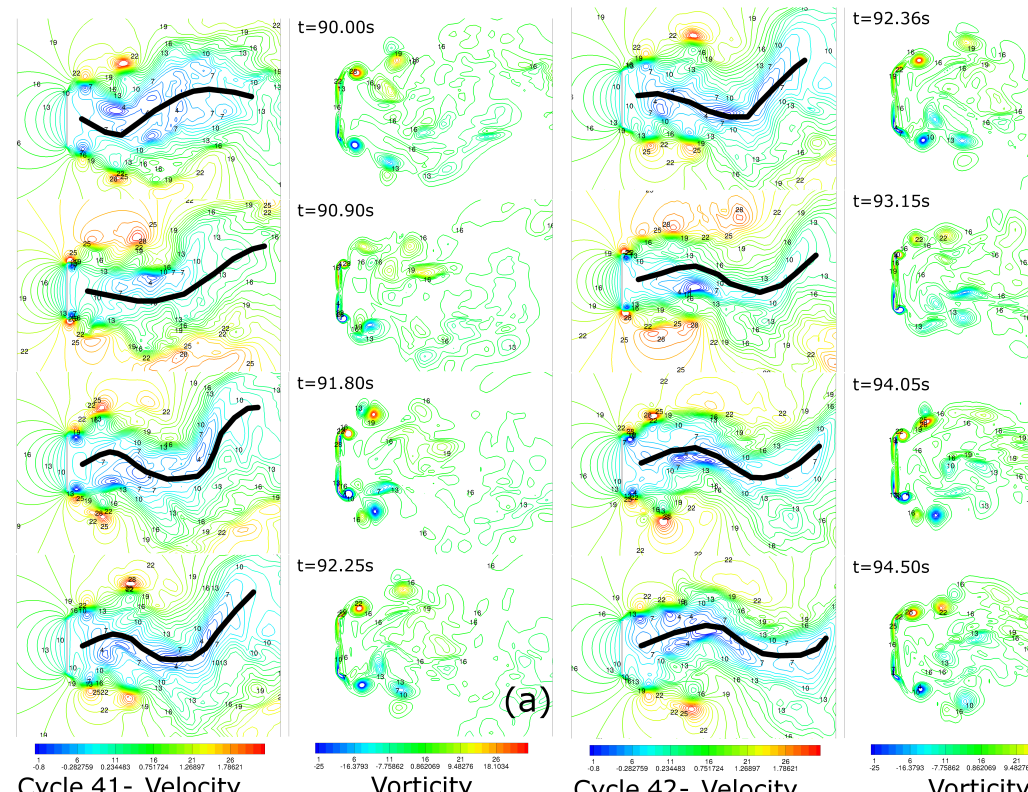

$t=93.15 \mathrm{~s}$
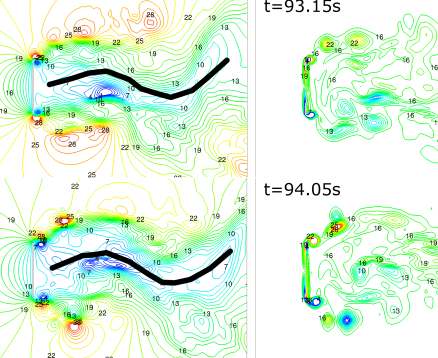

$t=94.05 \mathrm{~s}$

(a)
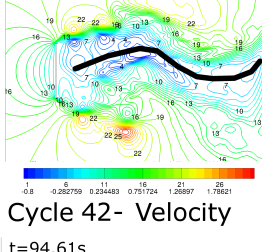

Cycle 41- Velocity

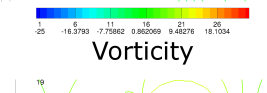
$\mathrm{t}=94.61 \mathrm{~s}$

Vorticity
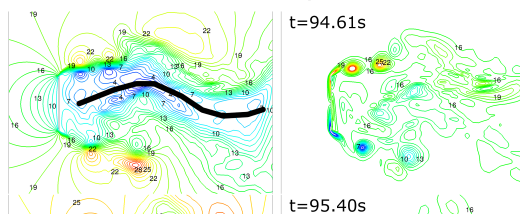

$\mathrm{t}=95.40 \mathrm{~s}$

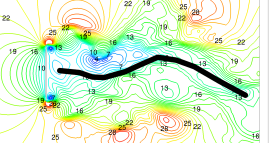

96.52
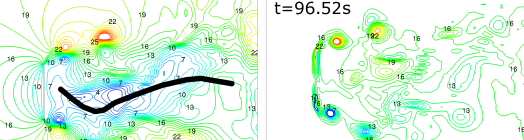

$t=94.50 \mathrm{~s}$
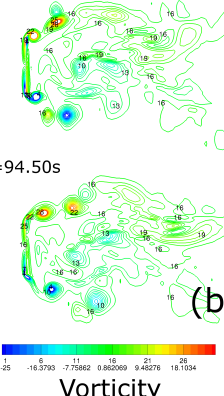